\title{
Pacific
}

Journal of

Mathematics

\section{HORIZONTAL PATH SPACES AND CARNOT-CARATHÉODORY} METRICS

\section{ZHONG GE}




\title{
HORIZONTAL PATH SPACES AND CARNOT-CARATHÉODORY METRICS
}

\section{ZHONG GE}

\begin{abstract}
In this paper we study a class of sub-spaces of loop spaces which have appeared in the calculus of variations. Generalizing a result of Smale, we show that the space of loops tangent to a distribution satisfying Hörmander's condition is weakly homotopic to the space of all loops. If the distribution is fat, we resolve the end point map from the space of horizontal paths. This resolution has two applications: (1) the proof that the cut-locus on an analytic fat Carnot-Caratheodory manifold is sub-analytic; (2) a study of the singularity of the horizontal loop space. At the end we study the geometry of left-invariant Carnot-Carathéodory metrics on fact nilpotent groups.
\end{abstract}

0. Introduction. In this paper we will study a class of sub-spaces of loop spaces which have appeared in the calculus of variations, control theorem (cf. [7], [12], [28]).

Let $M$ be a connected manifold, $H \subset T M$ a smooth distribution on $M$. We say that a $H^{1}$-curve $\gamma:[0,1] \rightarrow M$ is horizontal if it is tangent to $H$ almost everywhere. Let $\langle\cdot, \cdot\rangle$ be a fiberwise metric on $H$ (i.e. a Carnot-Carathéodory metric, or a $\mathrm{CC}$ metric for short), then the Carnot-Carathéodory distance (or a CC distance for short) on $M$ is defined to be

$$
d(x, y)=\inf E(\gamma)^{1 / 2}, \quad \text { where } E(\gamma)=\int_{0}^{1}\left\langle\frac{d \gamma(t)}{d t}, \frac{d \gamma(t)}{d t}\right\rangle d t .
$$

Here $\gamma$ runs over the space of horizontal paths connecting $x$ and $y$. A classical result of Chow's [9] says that if $H$ satisfies Hörmander's bracket generating condition, then every two points on $M$ can be joined by a horizontal path, so the distance is finite. The interest in horizontal loop spaces in part lies in the fact that they play a similar role in CC metrics as ordinary loop spaces play in Riemannian geometry.

There are many applications of $\mathrm{CC}$ metrics to Riemannian geometry, hypoelliptic operators, control theory, physics, etc. see [4], [6], [7], [14], [17], [18], [27], [30], [31], [33], [36], [38], [39], [40].

Let $\Omega_{H} M\left(x_{0}, \cdot\right)\left(\right.$ resp. $\left.\Omega_{H} M\left(x_{0}, x_{0}\right)\right)$ be the space of $H^{1}$-horizontal paths starting from $x_{0}$ (resp. $H^{1}$-horizontal loops based at $x_{0}$ ). 
We first look at the topology of $\Omega_{H} M\left(x_{0}, x_{0}\right)$. Smale proved that if $H$ is a contact distribution on a three dimensional manifold, then $\Omega_{H}\left(x_{0}, x_{0}\right)$ is weakly homotopic to the ordinary based loop space. In [12] we proved that $\pi_{0}\left(\Omega_{H}\left(x_{0}, x_{0}\right)\right)=\pi_{1}(M)$. In this paper we will prove

THEOREM 1. Let $M$ be a connected manifold with a bracket generating distribution $H$. Then the space of based horizontal loops, $\Omega_{H}\left(x_{0}, x_{0}\right)$ is weakly homotopic to the space $\Omega\left(x_{0}, x_{0}\right)$ of all loops: their homotopy groups are all equal. If, in addition the distribution is fat (see the definition in $§ 1.1$ ), then two spaces are homotopic.

In spite of Theorem 1 the two spaces are very different geometrically; for example, $\Omega_{H} M\left(x_{0}, x_{0}\right)$ is smooth, as the end point map $e: \Omega_{H}\left(x_{0}, \cdot\right) \rightarrow M, e(\gamma) \rightarrow \gamma(1)$ is not a submersion at the constant loop. We will focus our attention on the case of fat distributions, see Weinstein [41]. (Fat distributions are also called "strongly bracket generating" or "of maximal co-rank".) The following result is inspired by a result of Milnor [25] in finite dimensions:

THEOREM 2. If $H$ is a fat distribution, then the complement of the intersection of $\Omega_{H} M\left(x_{0}, x_{0}\right)$ with a sufficiently small sphere in $\Omega_{H} M\left(x_{0}, \cdot\right)$ at the constant loop is the total space of fibration over $S^{n-1}$ with contractible fibers, $n=\operatorname{dim}(M)$.

In contrast to the Riemannian case in which cut points cannot come arbitrarily close to $x_{0}$ and the complement of a cut loss is contractible [21], we prove

THEOREM 3. If $M$ is a fat CC manifold of dimension $n$, then the complement of the cut locus (see the definition in §5.1) of $x_{0}$ within any ball $d\left(x, x_{0}\right) \leq T$ is homeomorphic to the complement of a $k$-plane in $R^{n}$. Here $k=n-m$ is the codimension of the distribution.

Our final result concerns the structure of analytic CC manifolds.

THEOREM 4. Let $M$ be a fat analytic CC manifold. Then the cut locus of any point is sub-analytic within a sufficiently small neighborhood of that point.

There is a stronger version of this result, which says the ratio $d\left(x, x_{0}\right) / d_{1}\left(x, x_{0}\right)$, where $d_{1}\left(x, x_{0}\right)$ is the CC distance function on the tangent cone, is sub-analytic near $x_{0}$ (cf. Theorem 5.5). 
Sussmann [42] proved a result to which Theorem 4 is in a sense complementary. His result says that on a three-dimensional analytic CC manifold with a contact distribution, the cut locus of $x_{0}$ is subanalytic within any compact set of $M-\left\{x_{0}\right\}$.

Note that Theorem 3 and Theorem 4 together give us some information about how the cut-locus of $x_{0}$ within a small neighborhood of $x_{0}$ looks like, for example, that it is triangulable, and it has only one "branch". We conjecture that the cut locus within a sufficiently small neighborhood of $x_{0}$ can be stratified into analytic submanifolds of dimension $l$, where $k \leq l \leq n-1$.

The main technical tool used to prove Theorems 2 and 4 was introduced in the author's previous paper [12] in this journal and is of interest in its own right. This is the resolution of the end-point map $e: \Omega_{H} M\left(x_{0}, \cdot\right) \rightarrow M$, i.e. a submersion

$$
B_{e}: B \Omega_{H} M\left(x_{0}, \cdot\right) \rightarrow B_{w} M,
$$

where $B \Omega_{H} M\left(x_{0}, \cdot\right)$ is the blow-up of $\Omega_{H} M\left(x_{0}, \cdot\right), B_{w} M$ is the (weighted) blow-up of $M$ at $x_{0}$.

This paper is organized as follows. In $\S 1$ we recall the notion of tangent cones as developed by Folland, Rothschild, Stein, et al. In $\S 2$ we prove Theorem 1. In $\S 3$ we develop the main technical tool, i.e. the resolution of $e$. In $\S 4$ we study the singularity of the horizontal loop space. In $\S 5$ we study the cut-locus. In $\S 6$ we study left-invariant CC metrices on fat nilpotent Lie groups; in particular, we show that the set of fat nilpotent Lie algebras with $n, m$ fixed, $n-m=2$, is connected.

After the first version of this paper was written in the fall of 1989, Professor H. Sussman kindly pointed out some gaps in the proof of Theorem 4 in that version; in this version we have taken this into account. In fact we found a simpler method to prove Theorem 4 , but as the old method yields a stronger result (Theorem 5.5), we include both methods. Also Professor A. V. Sarychev kindly informed us that he has proved Theorem 1, which was announced in Dokl. Akad. Sci. USSR, v. 314(6), 1990.

A part of this work was done at the Mathematical Sciences Research Institute at Berkeley in 1988-89. We wish to thank Richard Montgomery for calling our attention to Smale's thesis, which is essential to Theorem 1, and many talks concerning CC metrics, Alan Weinstein for helpful conversations, H. Sussmann for pointing out some gaps in the first version of this manuscript, and finally, the 
referee for his many suggestions on how to improve the organization of this paper.

\section{Preliminary facts.}

1.1. Tangent cones. In this subsection we will recall some basic facts on Carnot-Carathéodory manifolds (or CC manifolds for short).

A Carnot-Carathéodory metric (or a CC metric) on a manifold $M$ is a symmetric bilinear semi-positive form $Q(\cdot, \cdot): T^{*} M \times T^{*} M \rightarrow R$. It induces a bundle homomorphism $L_{Q}: T^{*} M \rightarrow T M$ by

$$
\left\langle L_{Q}(p), q\right\rangle_{T M, T^{*} M}=Q(p, q), \quad p, q \in T^{*} M,
$$

where $\langle\cdot, \cdot\rangle_{T M, T^{*} M}$ is the natural pairing between $T M$ and $T^{*} M$. The image of $T^{*} M$ under $L_{Q}, H=L_{Q}\left(T^{*} M\right)$ is called the horizontal bundle, on which there is an induced metric

$$
(\cdot, \cdot): H \times H \rightarrow R \text {. }
$$

We always assume that $H$ is smooth.

We say that $H$ satisfies Hörmander bracket generating condition if we define

$$
H_{1}=H+[H, H], \ldots, H_{s}=H_{s-1}+\left[H_{s-1}, H_{s-1}\right], \ldots
$$

then for $s$ big enough, $H_{s}=T M . H$ is $s_{0}$-steps bracket generating if $s_{0}$ is the smallest integer such that $H_{s_{0}}=T M$. From now on we assume that $H$ satisfies Hörmander's condition unless specified otherwise.

A class of distributions of special interest to us is fat distributions. Recall that $H$ is fat if for any $a \in C^{\infty}(H), a \neq 0$, every vector can be locally written as $b_{1}+\left[a, b_{2}\right]$ for some $b_{1}, b_{2} \in C^{\infty}(H)$.

A rank $m$ fat distribution on an $n$ manifold must be a contact distribution if $m=n-1$; and $m$ must be a multiple of 4 if $n-m>1$ (cf. [32], Appendix 2).

LEMMA 1.1. If $H$ is fat, $x_{0} \in M$, then there are local coordinates $(x, y)$ at $x_{0}$ such that $H$ is locally defined by $\Lambda_{i}=0, i=1, \ldots, k$, where

$$
\Lambda_{i}=d y_{i}-\sum \Gamma_{j k}^{i} x_{j} d x_{k}+R_{i}, \quad \Gamma_{j k}^{i}=-\Gamma_{k j}^{i},
$$

and $R_{i}$ are 1-form satisfying

$$
\left|R_{i}\right| \leq M\left(|x|^{2}+|Y|\right) .
$$

A proof can be found in Goodman [14]. 
We now define a two-step nilpotent Lie algebra associated with $H$ at $x_{0}$, which plays a similar role as tangent spaces play in Riemannian geometry.

Definition 1.1. Using the notations in Lemma 1.1, we define a graded two-step nilpotent Lie algebra $n=V_{1} \oplus V_{2}$, where $\left[V_{1}, V_{1}\right] \rightarrow$ $V_{2}$ is given by

$$
\begin{aligned}
& {\left[\left(x_{1}^{1}, \ldots, x_{m}^{2}\right),\left(x_{1}^{2}, \ldots, x_{m}^{2}\right)\right]} \\
& \quad=\left(\sum \Gamma_{i j}^{1} x_{i}^{1} x_{j}^{2}, \ldots, \sum \Gamma_{i j}^{k} x_{i}^{1} x_{j}^{2}\right) .
\end{aligned}
$$

The simply connected Lie group of $n$ is called the tangent cone of $M$ at $x_{0}$.

Note that the bracket (1.1) can also be written in a coordinate-free way as follows

$$
H_{x_{0}} \times H_{x_{0}} \rightarrow T_{x_{0}} M / H_{x_{0}}, \quad \Omega\left(v_{1}, v_{2}\right)=\left[v_{1}, v_{2}\right] \bmod (H) .
$$

Obviously $H$ is fat if and only if the nilpotent algebra $n$ has the property that for any $a \in V_{1}, a \neq 0, \operatorname{ad}_{a}: V_{1} \rightarrow V_{2}$ is onto. We will call such two-step nilpotent Lie algebras fat nilpotent.

The construction of tangent cones can be generalized to higher order bracket generating distributions, due to Rothschild-Stein [33], see also Goodman [14].

1.2. Partial connections. To write down the geodesic equation for a CC metric, we will need the notation of partial connections.

Definition 1.2. Given a projection onto $H, \pi: T M \rightarrow H$, a map

$$
H_{x} \times C^{\infty}(H) \rightarrow C^{\infty}(H), \quad(u, v) \rightarrow D_{u} V
$$

depending smoothly on $x$, is a partial connection if

(1) it is linear in $\dot{u} \in H_{x}$, and for a function $f$ on $M$,

$$
D_{u}(f v)=u(f) v+f D_{u} v \text {. }
$$

$$
D_{v_{1}} v_{2}-D_{v_{2}} v_{1}=\pi\left[v_{1}, v_{2}\right]
$$

It is easy to prove that once $\pi$ is given, the partial connection exists uniquely. In fact, the partial connection can be given in the following way. We extend the CC metric to a Riemannian metric $g$ on $M$ such that $\pi$ becomes an orthogonal projection, and denote by $D^{g}$ the Levi-Civita connection on $g$; then the partial connection is given by

$$
D_{u} v=\pi D_{u}^{g} v
$$


Using these notations, we can easily write down the geodesic equations, which in local coordinates was derived in [39], p. 435, (12.1).

Suppose that a Riemannian metric $g$ is an extension of the $\mathrm{CC}$ metric, so we have an orthogonal decomposition $T M=H \oplus H^{\perp}$. Let $\gamma:[0,1] \rightarrow M$ be a CC geodesic; then it satisfies

$$
D_{\gamma^{\prime}} \gamma^{\prime}=\lambda \cdot\left(\gamma^{\prime}, \cdot\right)
$$

where $\Omega$ is as in (1.2) and $\lambda \in H^{\perp}$ is the Lagrange multiplier, which satisfies

$$
(I-\pi) D_{\gamma^{\prime}}^{g} \lambda=T\left(\gamma^{\prime}, \gamma^{\prime}\right),
$$

where $T: H \times H \rightarrow H^{\perp}$ is a tensor, whose exact form is not our concern in this paper.

2. Horizontal path space and horizontal loop space. In this section we will study the topology of the horizontal path space $\Omega_{H} M\left(x_{0}, \cdot\right)$ and the horizontal loop space $\Omega_{H} M\left(x_{0}, x_{0}\right)$.

LEMMA 2.1. $\Omega_{H} M\left(x_{0}, \cdot\right)$ is contractible.

LEMMA 2.2. $\Omega_{H} M\left(x_{0}, x_{0}\right)$ in the $C^{0}$-norm is a Hopf space.

These two lemmas can be proved as in the case of ordinary path spaces. Moreover, the above results are still true under the weaker hypothesis that every two points on $M$ can be joined by a horizontal path.

If $\gamma_{1}, \gamma_{2}$ are two horizontal paths, $\gamma_{1}(1)=\gamma_{2}(0)$, then $\gamma_{2} \cdot \gamma_{1}$ denotes the path defined by $\gamma(t)=\gamma_{1}(2 t), 0 \leq t \leq 1 ; \gamma(t)=$ $\gamma_{2}(2 t-1), 1 / 2 \leq t \leq 1$.

LEMMA 2.3. Every $x_{0} \in M$ has a neighborhood $N_{x_{0}}$ such that for any $x, y \in N_{x_{0}}$, there is a horizontal path $\gamma_{x y}$ within $N_{x_{0}}$ which joins $x$ to $y$ and depends continuously on $x, y$.

Proof. It suffices to prove that the following is true: for any $x \in$ $N_{x_{0}}$, there is a horizontal path $\gamma_{x}$ within $N_{x_{0}}$, joining $x_{0}$ to $x$ and depending continuously on $x$. In fact, if the above is true, let $\gamma_{x_{1} x_{2}}=$ $\gamma_{x_{2}} \cdot \gamma_{x_{1}}^{-1}$, then $\gamma_{x_{1} x_{2}}$ satisfies the requirement.

Suppose the vector fields $a_{1}, \ldots, a_{m}$ span $H$ in a neighborhood of $x_{0}$, and, together with their commutators,

$$
a_{J}=\left[a_{i_{1}}, \ldots,\left[a_{i_{r-1}}, a_{i_{r}}\right] \cdots\right], \quad J=\left(i_{1}, \ldots, i_{r}\right), \quad r \leq s
$$

$\operatorname{span} T_{x_{0}} M$. 
Choose $k$ multiple indices $J_{1}, \ldots, J_{k}$ such that

$$
a_{1}\left(x_{0}\right), \ldots, a_{m}\left(x_{0}\right), a_{J_{1}}\left(x_{0}\right), \ldots, a_{J_{k}}\left(x_{0}\right)
$$

are linearly independent and $\operatorname{span} T_{x_{0}} M$. Denote $J_{j}=\left(i_{1, j}, i_{2}, j, \ldots\right.$, $\left.i_{r_{j}, j}\right)$ and $\phi_{j}(t)=\exp \left(t a_{j}\right), j=1, \ldots, m$. We define inductively, for $J_{j}=\left(J_{1}, i_{j}\right)$,

$$
\begin{aligned}
\phi_{J_{i}}\left(t_{1}, \ldots, t_{j}\right)= & \phi_{J_{1}}\left(-\left|t_{1}\right|^{1 / 2}, \ldots,-\left|t_{j-1}\right|^{1 / 2}\right) \phi_{i_{j}}\left(-\left|t_{j}\right|^{1 / 2}\right) \\
& \phi_{J_{1}}\left(\left|t_{1}\right|^{1 / 2}, \ldots,\left|t_{j-1}\right|^{1 / 2}\right) \phi_{i_{j}}\left(\left|t_{j}\right|^{1 / 2}\right),
\end{aligned}
$$

if each $t_{0}>0$; otherwise, the right-hand side above should be changed to

$$
\begin{array}{r}
\phi_{i_{j}}\left(-\left|t_{j}\right|^{1 / 2}\right) \phi_{J_{1}}\left(-\left|t_{1}\right|^{1 / 2}, \ldots,-\left|t_{j-1}\right|\right)^{1 / 2} \\
\phi_{i_{j}}\left((|t|)^{1 / 2}\right) \phi_{J_{1}}\left(\left|t_{1}\right|^{1 / 2}, \ldots,\left|t_{j-1}\right|^{1 / 2}\right) .
\end{array}
$$

Then we have

$$
d \phi_{J_{i}}(t, t, \ldots, t) /\left.d t\right|_{t=0}=a_{J_{i}}
$$

Define

$$
\Phi\left(t_{1}, \ldots, t_{n}\right)=\phi_{J_{k}}\left(t_{1}, \ldots, t_{1}\right) \phi_{J_{k-1}}\left(t_{2}, \ldots, t_{2}\right) \cdots \phi_{1}\left(t_{n}\right) x_{0} .
$$

It is easy to verify that $\Phi$ is differentiable at 0 . Hence by the implicit function theorem and (2.1), $\Phi$ is a local diffeomorphism at $x_{0}$. We then define a horizontal path

$$
\begin{aligned}
& \gamma_{x}(t)=\phi_{J_{j}}\left(0, \ldots, 0,\left[r_{j}(n t-j+1)-(r-1)\right] t_{j}, t_{j}, \ldots, t_{j}\right) \\
& \quad \times \phi_{J_{k-1}}\left(t_{j+1}, \ldots, t_{j+1}\right) \cdots \phi_{1}\left(t_{n}\right) x_{0}, \\
& \frac{(j-1)}{n} \leq t \leq \frac{j}{n}, \quad \frac{(r-1)}{r_{j}} \leq n t-(j-1) \leq \frac{r}{r_{j}}, \quad j=1, \ldots, n ;
\end{aligned}
$$

where $\left(t_{1}, \ldots, t_{n}\right)=\Phi^{-1}(x)$, then this path joins $x_{0}$ to $x$ and depends continuously on $x$.

REMARK. Hörmander's condition is essential for the validity of Lemma 2.3. In fact, we can construct a distribution which does not satisfy Hörmander's condition, still any two points on $M$ can be joined by a piecewise smooth horizontal path, but for which Lemma 2.3 is not true: let $M=R^{3}$, and $H$ be spanned by $a_{1}, a_{2}$, where

$$
\begin{aligned}
& a_{1}= \begin{cases}\varphi\left(x_{1}\right) \partial / \partial x_{1}, & 0 \leq x_{1} \\
\varphi\left(-x_{1}\right)\left(\partial / \partial x_{2}-x_{1} \partial / \partial x_{3}\right), & x_{1} \leq 0 ;\end{cases} \\
& a_{2}= \begin{cases}0, & 0 \leq x_{1} \\
\varphi\left(-x_{1}\right)\left(\partial / \partial x_{1}+x_{2} \partial / \partial x_{3}\right), & x_{1} \leq 0 ;\end{cases}
\end{aligned}
$$

where $\varphi\left(x_{1}\right)$ is positive for $x_{1}>0$, and is zero for $x_{1} \leq 0$. 
THEOREM 2.4. $e: \Omega_{H} M\left(x_{0}, \cdot\right) \rightarrow M$ has the homotopy lifting property for polyhedra.

Proof. (We will use the notations in Lemma 2.3.) By Hurewicz's theorem, it suffices to prove that the map $e: e^{-1}\left(N_{x_{0}}\right) \rightarrow N_{x_{0}}$ has the lifting homotopy property for polyhedra (cf. [35]).

Let $P$ be a polyhedron, $h_{s}: P \rightarrow N_{x_{0}}, s \in[0,1]$ a given homotopy, $\mu: P \rightarrow e^{-1}\left(N_{x_{0}}\right)$ a map covering $h_{0}$. We construct a homotopy lifting $\mu_{s}: P \rightarrow e^{-1}\left(N_{x_{0}}\right)$ as follows.

Choose a continuous function $l, l(0)=0, l(s)>0$ if $s>0$, such that the path $t:[0,1-l(s)] \rightarrow(\mu(p))(t)$ is within $N_{x_{0}}$ for all $p \in P$, $s \in[0,1]$. Now define, for $p \in P$,

$$
\left(\mu_{s}(p)\right)(t)=\left\{\begin{array}{l}
(\mu(p))(t), \quad 0 \leq t \leq 1-l(s) ; \\
\gamma_{(\mu(p))(1-l(s)), h_{s}(p)}\left((t-1+l(s)) t(s)^{-1}\right), \quad 1-l(s) t \leq 1 .
\end{array}\right.
$$

So $\mu_{s}(p)$ first joins $(\mu(p))(0)$ to $(\mu(p))(1-l(s))$ along $\mu(p)$, then joins $(\mu(p))(1-l(s))$ to $h_{s}(p)$ along the horizontal path constructed in Lemma 2.3. By Lemma 2.3, $\mu_{s}(p)$ depends on $s, p$ continuously. Moreover, $e\left(\mu_{s}(p)\right)=h_{s}(p)$. Hence $e: e^{-1}\left(N_{x_{0}}\right) \rightarrow N_{x_{0}}$ is a fibration.

Proof of Theorem 1. Using the long exact sequence associated to the fibration $e$, we obtain $\pi_{k}\left(\Omega_{H} M\left(x_{0}, x_{0}\right)\right)=\pi_{k+1}(M)$.

If $H$ is fat, it is proved in [12] that the energy functional $E$ on $\Omega_{H} M\left(x_{1}, x_{2}\right)$ satisfies the Palais-Smale condition and is nondegenerate for generic $x_{2}$, so $\Omega_{H} M\left(x_{0}, x_{0}\right)$ is a CW-complex. By a theorem of Whitehead, a weak homotopy equivalence between two CW-complexes is a homotopy equivalence (cf. [35]), so $\Omega_{H} M\left(x_{0}, x_{0}\right)$ is homotopic to the ordinary based loop space.

As an application, we consider the case where $M$ is the total space of a principal $G$-bundle over $B: G \rightarrow M \stackrel{\pi}{\rightarrow} B$ with a connection $\omega_{0}$. Applying Theorem 1, we have

COROLlary 2.5. If at every point $x \in B$ the holonomy algebra is $g$ (the Lie algebra of $G$ ), then the space of based loops on $B$ with a given holonomy has the same homotopy groups as the based loop space does on $M$.

3. Resolution of end point map. Suppose that $H$ is fat. Then $x_{0}$ is an isolated singularity of the end point map $e$, and hence 
$\Omega_{H} M\left(x_{0}, x_{0}\right)$ is singular at the constant loop. In the next two sections we will study this singularity. In this section we will develop a resolution of $e$ by blowing up the source manifold (i.e. the horizontal paths space) and the target manifold $M$ simultaneously. We begin with the blow-up of $M$.

3.1. Weighted blow-up of $M$. We will use the notations in Lemma 1.1. We define the weighted blow-up of $M$ at $x_{0}$ to be $B_{w} M=$ $S_{1} \times[0, \infty)$, where $S_{1}$ is the set of points $\left(x_{1}, \ldots, x_{m}, y_{1}, \ldots, y_{k}\right)$ satisfying $x_{1}^{4}+\cdots+x_{m}^{4}+y_{1}^{2}+\cdots+y_{k}^{2}=1$. The blow-up map $B_{w}: B_{w} M \rightarrow M$ is given by

$$
\begin{aligned}
& B_{w}\left(\left(x_{1}, \ldots, x_{m}, y_{1}, \ldots, y_{k}\right), s\right) \\
& \quad=\left(s \cdot x_{1}, \ldots, s \cdot x_{m}, s^{2} \cdot y_{1}, \ldots, s^{2} \cdot y_{k}\right) .
\end{aligned}
$$

It is easy to see that $B_{w}$ is a diffeomorphism except at the boundary.

REMARK. A similar construction was developed by Kupka [44].

3.2. Blow-up of $\Omega_{H} M\left(x_{0}, \cdot\right)$. Let $\Omega R^{m}$ be the Hilbert space of $H^{1}$ paths on $R^{m}$ starting from $0 \in R^{m}$. As we will see $\S 3.3$, the horizontal path space $\Omega_{H} M\left(x_{0}, \cdot\right)$ in a neighborhood of the constant loop can be identified with $\Omega R^{m}$. From now on we will make this identification.

In view of this identification, we define the blow-up of $\Omega_{H} M\left(x_{0}, \cdot\right)$ to be $B \Omega_{H} M\left(x_{0}, \cdot\right)=S^{\infty} \times[0, \infty)$, where $S^{\infty}$ is the space of curves $\gamma$ on $R^{m}$ through $0,|\gamma|_{H^{1}}=1$. The blow-up map $B: B \Omega_{H} M\left(x_{0}, \cdot\right) \rightarrow$ $\Omega R^{m}$ is given by

$$
B(\gamma, s)=s \gamma .
$$

As in $\S 3.1$, it is easy to see that $B$ is a diffeomorphism except at the boundary.

3.3. Resolution of $e$. Having developed the blow-ups of the target and the source manifold of $e$, now we develop the resolution of $e$.

First we need a normal form of $e$. We begin with the simplest case: $M$ is a fat nilpotent group $N$ with the Lie algebra given by (1.1). In this case $\Omega_{H} M\left(x_{0}, \cdot\right)$ can be identified with the space of $H^{1}$-paths on $R^{m}$ starting from $0, \Omega R^{m}$, and $e$ can be written as

$$
\gamma \rightarrow\left(\gamma(1), \int_{\gamma} \omega_{1}, \ldots, \int_{\gamma} \omega_{k}\right),
$$

where

$$
\omega_{i}=\sum \Gamma_{j r}^{i} x_{j} d x_{r}, \quad i=1, \ldots, k
$$


For a general fat distribution, we will approximate $e$ using tangent cones. More precisely,

Lemma 3.1. Suppose that $H$ is a fat distribution and $N$ is the tangent cone at $x_{0}$. In a coordinate neighborhood of the constant loop, we can write $e$ as $e: \Omega R^{m} \rightarrow R^{n}$,

$$
\gamma_{1} \rightarrow\left(\gamma_{1}(1), \int_{\gamma_{1}} \omega_{1}+T_{1}\left(\gamma_{1}\right), \ldots, \int_{\gamma_{1}} \omega_{k}+T_{k}\left(\gamma_{1}\right)\right),
$$

where

$$
\left|T_{j}\left(\gamma_{1}\right)\right| \leq C\left\|\gamma_{1}\right\|^{3}, \quad j=1, \ldots, k
$$

Proof. Let $\gamma=\left(\gamma_{1}(t), y(t)\right)$ be a horizontal path; then we have the following equations: $\left(\gamma_{1}(t)=\left(x_{1}(t), \ldots, x_{m}(t)\right)\right)$

$$
\begin{aligned}
& \frac{d}{d t} y_{i}(t)=\sum \Gamma_{j r}^{i} x_{j}(t) \frac{d}{d t} x_{i}(t)+\bar{T}_{i}(x, y, \dot{x}, \dot{y}) \\
& i=1, \ldots, k
\end{aligned}
$$

where $\bar{T}_{i}(x, y, z, w)$ is a smooth function

$$
\left|\bar{T}_{i}(x, y, z, w)\right| \leq C_{0}\left(|x|^{3}+|y|^{2}\right),
$$

where $C_{0}$ is a uniform constant for bounded $z$.

It is obvious that given $\gamma_{1}(t) \in \Omega R^{m}$, there is a unique $\gamma=\left(\gamma_{1}(t)\right.$, $y(t)$ ) satisfying (3.3) and vice-versa, and the solution can be written as

$$
y_{i}(t)=\int_{\gamma_{1}} \omega_{i}+O\left(\|x\|^{3}+\|y\|^{2}\right) .
$$

Hence the lemma follows.

Let $\operatorname{gra}(e)$ be the graph of the restriction of $e$

$$
e: \Omega_{H} M\left(x_{0}, \cdot\right)-\Omega_{H} M\left(x_{0}, x_{0}\right) \rightarrow M-\left\{x_{0}\right\},
$$

and

$$
\left(B, B_{w}\right): B \Omega_{H} M\left(x_{0}, \cdot\right) \times B_{w}(M) \rightarrow \Omega_{H} M\left(x_{0}, \cdot\right) \times M
$$

be the obvious map.

Definition 3.1. The resolution

$$
B_{e}: B \Omega_{H} M\left(x_{0}, \cdot\right)-B^{-1}\left(\Omega_{H} M\left(x_{0}, x_{0}\right)\right) \rightarrow B_{w} M
$$

of $e$ is determined by its graph, which is the closure of $\left(B, B_{w}\right)^{-1}(\operatorname{gra}(e))$ in $B \Omega_{H} M\left(x_{0}, \cdot\right) \times B_{w}(M)$. 
The relation between various blow-ups can be summerized in the following diagram

$$
\begin{array}{cc}
B \Omega_{H} M\left(x_{0}, \cdot\right)-B^{-1}\left(\Omega_{H} M\left(x_{0}, x_{0}\right)\right) & \stackrel{B_{e}}{\longrightarrow} B_{w} M \\
\downarrow_{B} & \downarrow^{B_{w}} \\
\Omega_{H} M\left(x_{0}, \cdot\right)-\Omega_{H} M\left(x_{0}, x_{0}\right) & \stackrel{e}{\longrightarrow} M
\end{array}
$$

LEMMA 3.2. $B_{e}$ is a submersion everywhere.

Proof. By (3.4), since both $B_{w}$ and $B$ are diffeomorphisms except at the boundaries, we need only to prove that $B_{e}$ is submersive at the boundary of $B \Omega_{H} M\left(x_{0}, \cdot\right)$.

Using Lemma 3.1 and (3.1), (3.2), we see that $B_{e}$ can be written as

$$
\begin{array}{r}
\left(\gamma_{1}, s\right) \rightarrow\left(\left(\frac{1}{b} s \gamma_{1}(1), \frac{1}{b^{2}}\left(\int_{s \gamma_{1}} \omega_{1}+T_{k}\left(s \gamma_{1}\right)\right), \ldots,\right.\right. \\
\left.\left.\frac{1}{b^{2}}\left(\int_{s \gamma_{1}} \omega_{k}+T_{k}\left(s \gamma_{1}\right)\right)\right), b\right),
\end{array}
$$

where

$$
\begin{aligned}
b=\left(s^{4}\left(x_{1}^{4}+\cdots+x_{m}^{4}\right)+\left(\int_{s \gamma_{1}} \omega_{1}+T_{k}\left(s \gamma_{1}\right)\right)^{2}\right. & \\
& \left.+\cdots+\left(\int_{s \gamma_{1}} \omega_{k}+T_{k}\left(s \gamma_{1}\right)\right)^{2}\right)^{1 / 4}
\end{aligned}
$$

for $\left(x_{1}, \ldots, x_{m}\right)=\gamma_{1}(1)$. By (3.3), we can rewrite (3.5) as

$$
\begin{aligned}
B_{e}\left(\gamma_{1}, s\right)= & \left(\left(\gamma_{1}(1), \int_{\gamma_{1}} \omega_{1}, \ldots, \int_{\gamma_{1}} \omega_{k}\right),\right. \\
& \left.s\left(x_{1}^{4}+\cdots+x_{k}^{4}+\left(\int_{\gamma_{1}} \omega_{1}\right)^{2}+\cdots+\left(\int_{\gamma_{1}} \omega_{k}\right)^{2}\right)^{1 / 4}\right)+o(s) .
\end{aligned}
$$

So it is a submersion.

4. Singularity of horizontal loop space. In this section we will study the singularity of the horizonal loop space, using the resolution of $e$. 
4.1. The case of nilpotent groups. Suppose that $M$ is a simple connected nilpotent Lie group $N$ whose Lie algebra is

$$
\begin{gathered}
n=V_{1} \oplus V_{2} \oplus \cdots \oplus V_{s}, \quad\left[V_{i}, V_{j}\right] \subset V_{i+j}, \quad i+j \leq s, \\
{\left[V_{i}, V_{j}\right]=0, i+j>s,}
\end{gathered}
$$

and $H$ is the left translation of $V_{1}$. As usual, we denote the space of $H^{1}$ horizontal paths starting from 0 by $\Omega_{H} N(0, \cdot)$.

$R_{+}$(the multiplicative group of positive numbers) acts on $N-\{0\}$ as

$$
t\left(a_{1}, a_{2}, \ldots, a_{n}\right)=\left(a_{1} t, \ldots, a_{m} t, a_{m+1} t^{2}, \ldots, a_{i_{k}} t^{k}, \ldots, a_{n} t^{s}\right),
$$

where $a_{i_{k}} \in V_{k}$. Let $P_{w} N$ be the quotient space. It is easy to prove that $P_{w}$ is diffeomorphic to $S^{n-1}, n=\operatorname{dim}(N)$.

The action of $R_{+}$on $N$ induces an action on $\Omega_{H} N(0, \cdot)$, $\Omega_{H} N(0,0)$ respectively. Let $P\left(\Omega_{H} N(0, \cdot)-\Omega_{H} N(0,0)\right)$ be the quotient of the space $\Omega_{H} N(0, \cdot)-\Omega_{H} N(0,0)$ by this action. Then the end point map $e$ induces a map

$$
\{e\}: P\left(\Omega_{H} N(0, \cdot)-\Omega_{H} N(0,0)\right) \rightarrow P_{w} N
$$

in an obvious way.

THEOREM 4.1. $\{e\}$ is a fibration over $S^{n-1}$.

Proof. We only need to prove an equivariant version of Lemma 2.3, i.e. there is $\delta_{0}>0$, such that for any $x, y, d(x, y) \leq \delta_{0}$, there is a horizontal curve $\gamma_{x, y}$ connecting $x, y$, depending continuously on $x, y$, and

$$
\gamma_{s x, s y}=s \cdot \gamma_{x, y}
$$

where $S \cdot \gamma$ denotes the induced action of $R_{+}$on $\Omega_{H} N(0, \cdot)$.

In fact, there are left-invariant vector fields on $N, a_{1}, \ldots, a_{m} \in$ $\Gamma(H)$ spanning $H$, and, together with their commutators of length less than $(s+1), a_{J}=\left[a_{i_{1}}, \ldots,\left[a_{i_{r-1}}, a_{i_{r}}\right]\right], J=\left(i_{1}, \ldots, i_{r}\right), r \leq s$ span $T N$. We then define $\Phi$ in the same way as in Lemma 2.3.

To construct $\gamma_{x, y}$, we need to prove that $\Phi$ is equivariant, i.e.

$$
\begin{aligned}
& \Phi\left(s\left(t_{1}, \ldots, t_{n}\right)\right)\left(s\left(x_{1}, \ldots, x_{n}\right)\right) \\
& \quad=s \cdot \Phi\left(t_{1}, \ldots, t_{n}\right)\left(x_{1}, \ldots, x_{n}\right) .
\end{aligned}
$$

To this end, we need the notation of (weighted)-homogeneity.

A function $f$ on $N$ is homogeneous of degree $k$ if

$$
f\left(s\left(x_{1}, \ldots, x_{n}\right)\right)=s^{k} f\left(x_{1}, \ldots, x_{n}\right) .
$$


A vector field $V$ on $N$ is homogeneous of order 1 if for every homogeneous function $f$ of degree $k, V(f)$ is homogeneous of degree $(k-1)$. It is easy to prove that $a_{1}, \ldots, a_{m}$ are homogeneous of degree 1 , so (4.1) follows. The rest of the proof is the same as in Theorem 2.4.

REMARK 1. The fibre of the above fibration is contractible. In fact, the fibre is just $e^{-1}\left(x_{0}\right), x_{0} \neq 0$, which, by a result of Weinstein (cf. [12], Lemma 2.1), is homotopic to $e^{-1}(0)$, which is contractible.

REMARK 2. Let $\Omega R^{n}(0, \cdot)$ be the space of $H^{1}$-curves on $R^{n}$ through $0, e_{1}$ the end point map, and $\Omega R^{n}(0,0)=e_{1}^{-1}(0)$. $R_{+}$acts on $R^{n}-\{0\}$ in an obvious way and hence on $\Omega R^{n}(0, \cdot)-$ $\Omega R^{n}(0,0)$. Let $P_{+} R^{n}, P_{+}\left(\Omega R^{n}(0, \cdot)-\Omega R^{n}(0,0)\right)$ be the quotient spaces respectively; then as in Theorem 4.1 , the induced map $\left\{e_{1}\right\}$ : $P_{+}\left(\Omega R^{n}(0, \cdot)-\Omega R^{n}(0,0)\right) \rightarrow P_{+} R^{n}$ is a fibration with contractible fiber. It would be interesting to compare this fibration with the fibration in Theorem 4.1.

4.2. The case of general fat distributions. In this subsection we will generalize the results in $\S 4.1$ to a general fat distribution $H$. This generalization is not straight forward, as the map $e$ is not stable at the constant loop.

Let $N$ be the tangent cone at $x_{0} \in M$, and

$$
\begin{aligned}
& B\left(\Omega_{H} M\left(x_{0}, \cdot\right)-\Omega_{H} M\left(x_{0}, x_{0}\right)\right) \\
& \quad=B \Omega_{H} M\left(x_{0}, \cdot\right)-B^{-1}\left(\Omega_{H} M\left(x_{0}, x_{0}\right)\right),
\end{aligned}
$$

which can be considered as the blow-up of $\Omega_{H} M\left(x_{0}, \cdot\right)-\Omega_{H} M\left(x_{0}, x_{0}\right)$. Note that the boundary of $B\left(\Omega_{H} M\left(x_{0}, \cdot\right)-\Omega_{H} M\left(x_{0}, x_{0}\right)\right)$ is just $P\left(\Omega_{H} N(0, \cdot)-\Omega_{H} N(0,0)\right)$, which is a fibration over $S^{n-1}$ by Theorem 4.1 .

We define a Riemannian structure on $\Omega R^{n}$ by

$$
\left(\gamma_{1}, \gamma\right)_{1}=\int_{0}^{1}\left(\frac{d \gamma(t)}{d t}, \frac{d \gamma(t)}{d t}\right) d t, \quad\|\gamma\|=(\gamma, \gamma)_{1}^{1 / 2},
$$

where $(\cdot, \cdot)$ is the Euclid structure on $R^{m}$. Also denote

$$
D^{-1} \gamma(t)=\int_{0}^{t} \gamma(s) d s
$$

To be precise about the sphere in Theorem 2, we need to introduce a function on $\Omega R^{m}$. 
Write $e=\left(e_{1}, \ldots, e_{n}\right)$. Let $d e_{i}$ be the differential of the functional $e_{i}$. Then $D^{-2} d e_{i}$ formally is the gradient of the functional $e_{i}$ with respect to $(\cdot, \cdot)_{1}$.

We define a function $\Omega R^{m}$

$$
\begin{aligned}
P(\gamma)=\operatorname{det}\left(D^{-2} d e_{i}(\gamma)(t)-t D^{-2} d e_{i}(\gamma)(1),\right. & \left.\left(D^{-2} d e_{i}(\gamma)(t)-t D^{-2} d e_{i}(\gamma)(1)\right)\right) .
\end{aligned}
$$

Since $H$ is fat, $P(\gamma) \neq 0$ if $\gamma \neq 0$.

Now we define a sphere

$$
S_{\varepsilon}=\left\{\gamma \in \Omega R^{m},\|\gamma\|^{1 / 2}+\|\gamma\| / P(\gamma)=\varepsilon\right\} .
$$

Now we reformulate Theorem 2 as follows:

THEOREM 4.2. Let $S_{e}=\Omega_{H} M\left(x_{0}, x_{0}\right) \cap S_{\varepsilon}$. Then the complement of $S_{e}$ in $S_{\varepsilon}$ is the total space of a fibration over $S^{n}$ with contractible fiber for $\varepsilon$ small enough.

REMARK. We do not know if the theorem will remain true if we replace $S_{\varepsilon}$ by the standard sphere.

4.3. The proof of Theorem 4.2. The basic idea of the proof is to study a vector field (which we call $F$ ) on $B \Omega_{H} M\left(x_{0}, \cdot\right)$. We will prove that its phase flow induces a homeomorphism from $S_{\varepsilon}-S_{e}$ to $P\left(\Omega_{H} N(0, \cdot)-\Omega_{H} N(0,0)\right)$, which is, by Theorem 4.1 , a fibration over $S^{n-1}$.

We first construct a vector field on $\Omega R^{m}$,

$$
\frac{d \gamma_{s}(t)}{d s}=-\gamma_{s}(t)+\sum a_{i}\left(\gamma_{s}\right)\left(D^{-2} d e_{i}\left(\gamma_{s}\right)(t)-D^{-2} d e_{i}\left(\gamma_{s}\right)(1)\right),
$$

where $a_{1}(\gamma), \ldots, a_{k}(\gamma)$ satisfy

$$
\begin{aligned}
& \sum a_{i}(\gamma)\left(D^{-2} d e_{i}(\gamma)-t D^{-2} d e_{i}(\gamma)(1),\right.\left.D^{-2} d e_{j}(\gamma)(t)-t D^{-2} d e_{j}(\gamma)(1)\right)_{1} \\
&=\left(D^{-2} d e_{j}(\gamma)(t)-t^{2} d e_{j}(\gamma)(1), \gamma\right)_{1}-2 e_{j}(\gamma), \\
& j=1, \ldots, k .
\end{aligned}
$$

This condition is to assure that the vector field (4.2) leaves the set $e^{-1}(0)$ invariant. Note that when restricted to $e^{-1}(0),(4.2)$ is just the gradient flow of the functional $\|\gamma\|^{2}$ with respect to the Riemannian product $(\cdot, \cdot)_{1}$. 
Solve $a_{1}, \ldots, a_{k}$ from (4.3); then we can rewrite (4.2) as

$$
\frac{d \gamma(s, t)}{d s}=-\gamma(s, t)+\frac{V\left(\gamma_{s}\right)(t)}{P\left(\gamma_{s}\right)}
$$

where $V, P$ satisfy the following conditions ( $C$ will denote any constant)

(1) $P$ is a smooth function on $\Omega R^{m}, P(\gamma)=P_{0}(\gamma)+P_{1}(\gamma)$, where $P_{0}$ is a positive homogeneous functional of degree $2 k$, and

$$
\left|P_{1}(\gamma)\right| \leq C\|\gamma\|^{2 k+1}
$$

(2) $V$ is a smooth vector field on $\Omega R^{m}$

$$
\|V(\gamma)\| \leq C\|\gamma\|^{2 k+2} \text {. }
$$

We will use $d / d s$ to denote the Lie derivative with respect to the vector field (4.2). Using (4.5), (4.6), by a direct computation we obtain

$$
\begin{aligned}
\frac{d}{d s}\left(\frac{\|\gamma\|^{2 k+1 / 2}}{P(\gamma)}\right) \leq & -\frac{\|\gamma\|^{2 k+1 / 2}}{2 P}+C \frac{\|\gamma\|^{4 k+3 / 2}}{P^{2}} \\
& +C \frac{\|\gamma\|^{6 k+3 / 2}}{P^{3}}(1+\|\gamma\|) .
\end{aligned}
$$

This will be our fundamental inequality. As we will see, if $\|\gamma\|$ and $\|\gamma\|^{2 k+1 / 2} / P(\gamma)$ are sufficiently small, then they are fast decreasing.

LEMMA 4.3. If

$$
\frac{\left\|\gamma_{0}\right\|}{P\left(\gamma_{0}\right)}\left(\left\|\gamma_{0}\right\|^{1 / 2}+\frac{\left\|\gamma_{0}\right\|}{P\left(\gamma_{0}\right)}\left(1+\left\|\gamma_{0}\right\|\right)\right) \leq c \leq 1,
$$

where $c$ is sufficiently small, then there is a constant $c_{1}, 0<c_{1}<1$,

$$
\frac{\left\|\gamma_{s}\right\|^{2 k+1 / 2}}{P\left(\gamma_{s}\right)} \leq \frac{\left\|\gamma_{0}\right\|^{2 k+1 / 2}}{P\left(\gamma_{0}\right)} \exp \left(\left(-1+c_{1}\right) s\right) \text {. }
$$

Proof. Applying the Gronwall inequality to (4.7).

CoROllaRy 4.4. Under the same condition in Lemma 4.3, there is a constant $c_{2}, 0<c_{2}<1 / 6$, such that

$$
\left\|\gamma_{s}\right\| \leq \exp \left(-s+s c_{2}\right)\left\|\gamma_{0}\right\|
$$

Proof. Using (4.4), we have

$$
\frac{d}{d s}\|\gamma\|=-\|\gamma\|+\frac{(\gamma, V(\gamma))_{1}}{\|\gamma\| P(\gamma)} \leq-\|\gamma\|+\frac{\|\gamma\|^{2 k+1}}{\|\gamma\| P(\gamma)} \leq-\|\gamma\|\left(1-C c^{2}\right) \text {. }
$$


Using the Gronwall inequality, we prove the lemma.

So far we have proved that the phase flow of (4.2) will approach 0 within $S_{e}$ very fast, so 0 is a singularity of this vector field. To resolve this singularity, we will pull back this vector field by $B$ to $B \Omega R^{m}$ and then divide it by $|\gamma|$. The vector field $F$ thus obtained will induce a homeomorphism $S_{\varepsilon}-S_{e} \rightarrow P\left(\Omega_{H} N(0, \cdot)-\Omega_{H} N(0,0)\right)$.

To be more precise, define $\beta_{s}=\gamma_{s} /\left\|\gamma_{s}\right\|$; then $\beta_{s}$ satisfies the following equation:

$$
\frac{d \beta_{s}}{d s}=-\frac{\left(V\left(\gamma_{s}\right), \gamma_{s}\right)_{1} \beta_{s}}{\left\|\gamma_{s}\right\|^{2} P\left(\gamma_{s}\right)}+\frac{V\left(\gamma_{s}\right)}{\left\|\gamma_{s}\right\| P\left(\gamma_{s}\right)}
$$

which, together with the equation for $\left\|\gamma_{s}\right\|$,

$$
\frac{d\left\|\gamma_{s}\right\|}{d s}=-\left\|\gamma_{s}\right\|+\frac{\left(V\left(\gamma_{s}\right), \gamma_{s}\right)}{\left\|\gamma_{s}\right\| P\left(\gamma_{s}\right)}
$$

is the pull back of (4.2). Obviously this vector field vanishes on the boundary of $B \Omega R^{m}$.

Note that $\beta_{s}$ converges as $s \rightarrow \infty$. To see this, we estimate $d \beta_{s} / d s$. From (4.10), we have

$$
\left\|\frac{d}{d s} \beta_{s}\right\| \leq C \frac{\left\|\gamma_{s}\right\|^{2 k+1}}{P\left(\gamma_{s}\right)},
$$

then, using Lemma 4.3, Corollary 4.4, we have

$$
\left\|\beta_{s}-\beta_{0}\right\| \leq C \frac{\left\|\gamma_{0}\right\|^{2 k+1}}{P\left(\gamma_{0}\right)} .
$$

The vector field $F$ is then obtained by dividing (4.10), (4.11) by $\|\gamma\|$

$$
\begin{gathered}
\frac{d \beta_{s}}{d s}=-\frac{\left(V\left(\gamma_{s}\right), \gamma_{s}\right)_{1} \beta_{s}}{\|\gamma\|^{3} P\left(\gamma_{s}\right)}+\frac{V\left(\gamma_{s}\right)}{\left\|\gamma_{s}\right\|^{2} P\left(\gamma_{s}\right)}, \\
\frac{d\left\|\gamma_{s}\right\|}{d s}=-1+\frac{\left(V\left(\gamma_{s}\right), \gamma_{s}\right)}{\left\|\gamma_{s}\right\|^{2} P\left(\gamma_{s}\right)} .
\end{gathered}
$$

Obviously $F$ is a smooth vector field on $S^{\infty} \times[0, \infty)$, nonvanishing up to the boundary. Note that by (12), the phase flow of $F$ determines a continuous map $S_{e} \rightarrow S^{\infty}$. Moreover, using a standard argument in the theory of smooth vector fields on manifolds and the fact that $F$ is non-vanishing, we then prove that this map is in fact a homeomorphism, so 
LEMMA 4.5. The phase flow of $F$ induces a homeomorphism $S_{\varepsilon}-$ $S_{e} \rightarrow P\left(\Omega_{H} N(0, \cdot)-\Omega_{H} N(0,0)\right)$.

5. Cut-locus of $\mathbf{C C}$ metrics. In this section we will study the cut locus of a CC metric. We will prove Theorem 3 in $\S 5.1$, Theorem 4 in $\S 5.2$.

5.1. Geometry and definition of the cut locus. Let $M$ be a complete CC manifold. Geodesics and minimizing geodesics are defined as in Riemannian geometry. If $H$ is fat, then the geodesics satisfy the geodesic equation ([12], compare [28] for the general case). $\exp _{x_{0}}: T_{x_{0}}^{*} M \rightarrow M$, or simply exp will denote the exponential map for the CC metric (cf. [12]). Thus exp is the composition of the time 1 map of the Hamiltonian flow on $T^{*} M$ composed with the inclusion of $T_{x_{0}}^{*} M$ into $T^{*} M$, projected to $M$.

Fix a point $x_{0} \in M$. Then any geodesic through $x_{0}$ has the form $\gamma(t)=\exp (t p)$. A "cut point" of $x_{0}$ along $\gamma$ is a point $x_{1}=\gamma\left(t_{1}\right)$ lying on $\gamma$ such that $\left[x_{0}, x_{1}\right]$ is the largest subinterval of $\gamma$ for which the geodesic is minimizing. In other words, if $t>t_{1}$ then $\gamma[0, t]$ is not a minimizing geodesic, and if $t \leq t_{1}$ then it is a minimizing geodesic. The set of all cut points (as $\gamma$ varies) to $x_{0}$ is called the cut locus (of $x_{0}$ ).

Note that $x_{1}$ is a cut point of $x_{0}$ if and only if either (a) there is more than one minimizing geodesics connecting $x_{0}$ to $x_{1}$ or (b) we can write $x_{1}=\exp (p)$ where $p$ is a critical point of $\exp$, and if $t<1$ then $t p$ is not a critical point. Note that (a) and (b) are not mutually exclusive. The points satisfying (b) are said to be in the "conjugate locus". Strictly speaking, the critical values of exp form the conjugate locus and the set of points satisfying (b) are said to be in the "first conjugate locus". For more on the cut locus and conjugate locus see Taylor [38], Lemma 5, or Kobayashi-Momizu [43], vol. 2, §8.7.

Strichartz [36] proved that cut points of $x_{0}$ occur arbitrarily near $x_{0}$. In fact conjugate points occur arbitrarily near $x_{0}$ (cf. [12]) and 0 is a critical point for exp with corresponding value $x_{0}$. For these reasons we now define $x_{0}$ to be in its own cut locus. Thus, for our purpose the cut locus is the usual cut locus, with $x_{0}$ included.

If $H$ is strong bracket generating, a geodesic does not minimize past its first conjugate point. It follows from this that (after adding $x_{0}$ in!) the cut locus is closed. Taylor (see [38], Theorem 4) claimed that a geodesic does not minimize past its first conjugate point, but we find a counter-example, see the Remark 2 after the proof of Theorem 3 . 
Let $S$ be the unit cylinder $Q(p, p)=1$ in $T_{x_{0}}^{*} M$. Let $\mu=\mu_{x_{0}}: S \rightarrow$ $R^{+}$be the function which assigns to each $p \in S_{x}$ the largest extended real number $T \in[0, \infty]$ such that the geodesic $t \rightarrow \exp _{x_{0}}(t p)$ is minimizing for all $t \in(0, T]$. Taylor [38] proved that if $\mu$ is locally bounded away from zero and $H$ is fat, then $\mu$ is continuous. He also conjectured that $\mu$ is continuous. Here we will prove his conjecture.

LEMMA 5.1. $\mu$ is locally bounded from below away from zero.

Proof. By the result of Rayner [32], for any CC geodesic $\gamma:[a, b] \rightarrow$ $M$ without double points, there is a Riemannian metric which extends the CC metric, with respect to which $\gamma$ is still a geodesic. Moreover, this Riemannian metric depends smoothly on $\gamma$. Obviously, if $\gamma$ minimizes the Riemannian action then it also minimizes the $\mathrm{CC}$ action. Since for a family of smoothly varying Riemannian metrics the injectivity radii are locally bounded away from zero, so is $\mu$.

Proof of Theorem 3. For $r>0$, let $B_{r}$ be the ball at $x_{0}, d\left(x, x_{0}\right) \leq$ $r$. We want to prove that the complement of the cut locus within $B_{r}$ is homeomorphic to the complement of a $k$-plane in $R^{n}$.

Let $D$ be the set of points $p$ in $T_{x_{0}}^{*} M$ such that the geodesic $t \rightarrow$ $\gamma(t)=\exp (t p)$ is minimizing for $0<t \leq 1, \exp (p) \in B_{r}$, and $\exp (p)$ is not a cut point to $x_{0}$. We first prove that $D$ is homeomorphic to the complement of $R^{k}$ in $R^{n}$. This follows from the following properties of $D$ :

(1) $D$ is open (since the cut-locus is closed).

(2) If $p \in D$, then $t p \in D$ for $0<t<1$. In fact, if $\exp _{x_{0}}\left(t_{0} p\right)$ is a cut point for some $t_{0} \in(0,1)$, then there is another geodesic $\gamma_{0}$ joining $\exp _{x_{0}}\left(t_{0} p\right)$ to $x_{0}$ with length less or equal to that of $t \rightarrow$ $\exp _{x_{0}}(t p)$; hence if $\gamma_{1}$ denotes the curve $\gamma_{1}=\gamma_{0}(2 t)$, if $0 \leq t \leq 1 / 2$; $\gamma_{1}(t)=\exp _{x_{0}}\left(\left(2(1-t) t_{0}+(2 t-1)\right) p\right)$, if $1 / \leq t \leq 1$, then $\gamma_{1}$ is also minimizing as it has the same length as $\gamma_{0}$, and hence must be smooth (as every minimizing geodesic is smooth), which is a contradiction.

(3) If $Q(p, p) \neq 0$, then for $t$ sufficiently small, $t p \in D$. This follows from $\mu_{x_{0}}>0$.

(4) If $Q(p, p)=0$, then $\exp (p)=x_{0}$.

Let $C\left(x_{0}\right)$ be the cut locus of $x_{0}$. Then we prove that exp: $D \rightarrow$ $M-C\left(x_{0}\right) \cap B_{r}$ is a homeomorphism. Obviously $\exp _{x_{0}}: D \rightarrow M-$ $C\left(x_{0}\right) \cap B_{r}$ is a local diffeomorphism. We need only to prove that it is one-to-one. 
Suppose that $p_{1}, p_{2} \in D, p_{1} \neq p_{2}, \exp _{x_{0}}\left(p_{1}\right)=\exp _{x_{0}}\left(p_{2}\right)$. Choose $t_{1}>1$ such that $t_{1} p_{2} \in D$. Define $\gamma_{3}(t)=\exp \left(2 t p_{1}\right)$, if $0 \leq t \leq 1 / 2$, and $\gamma_{3}(t)=\exp \left(\left(\left((2 t-1) t_{1}+2-2 t\right) p_{2}\right)\right)$ if $1 / 2 \leq t \leq 1$, then $\gamma_{3}$ is also minimizing (since it has the same length as the path $t:\left[0, t_{1}\right] \rightarrow$ $\left.\exp \left(t p_{2}\right)\right)$, and hence must be smooth, which is a contradiction.

REMARK 1. There is no such simple relation between the topologies of the cut locus and $M$ as in Riemannian geometry (cf. [21]).

REMARK 2. Theorem 3 is not true in general, as the following example shows. Take $M=R^{5}, H$ spanned by

$$
\frac{\partial}{\partial x_{1}}, \quad \frac{\partial}{\partial y_{1}}, \quad \frac{\partial}{\partial x_{2}}-y_{2} \frac{\partial}{\partial z_{1}}, \quad \frac{\partial}{\partial y_{2}}+x_{2} \frac{\partial}{\partial z_{1}},
$$

and the metric

$$
\left(d x_{1}\right)^{2}+\left(d y_{1}\right)^{2}+\left(d x_{2}-y_{2} d z_{1}\right)^{2}+\left(d y_{2}+x_{2} d z_{1}\right)^{2},
$$

then the cut-locus of 0 is

$$
\begin{gathered}
\left\{\left(x_{1}, y_{1}, x_{2}, y_{2}, z_{1}\right) \in R^{5}, x_{2}=y_{2}=0\right\} \\
-\left\{\left(x_{1}, y_{1}, 0,0,0\right),\left(x_{1}, y_{1}\right) \neq 0\right\} .
\end{gathered}
$$

In particular, the cut locus is not closed. The geodesic $t \rightarrow\left(p_{1} t, p_{2} t\right.$, $0,0,0)$ is minimizing within $[0, \infty)$, though every point on it is a critical yalue of the exponential map.

5.2. Cut-locus on fat analytic CC manifolds. We will prove Theorem 4. Let $M$ be a fat analytic CC manifold. We will need the following characterization of the cut-locus.

LEMMA 5.2. The cut-locus of $x_{0}$ is the singular support of the function $d\left(x, x_{0}\right)$. (The singular support of a function is the set of points where the function is not analytic, $c f .[37]$.

Proof. To see that this is in fact equivalent to our definition apply the inverse function theorem to exp to conclude that if $x$ is not in the cut locus then it is not in this singular support of $d\left(\cdot, x_{0}\right)$. (Note that, according to our definition of cut locus, if $x$ is not in the cut locus and if $\gamma=\exp (t p)$ is a minimizing geodesic joining $x_{0}$ to $x=\exp (p)$, then $p$ is not a critical point of exp.) Conversely, if $x$ is in the cut locus, then it must be in the singular support. Join $x$ to $x_{0}$ by a geodesic $\gamma$ as in the definition. Without loss of generality, we may take $\gamma$ to be unit speed. Suppose that $x=\gamma\left(t_{1}\right)$. Now observe that 
$d\left(\gamma(t), x_{0}\right)=t$ for $t \leq t_{1}$ but that $d\left(\gamma(t), x_{0}\right)<t$ for $t>t_{1}$. Since $\gamma$ is analytic it follows that $d$ cannot be analytic at $x$.

Now it suffices to prove that the distance $d\left(x, x_{0}\right)$ is sub-analytic in $x$ near $x_{0}$ since the singular support of a sub-analytic function is sub-analytic.

TheOREM 5.3. Suppose that the CC metric is real analytic and $H$ is fat; then there exists $T>0$ such that $d\left(x, x_{0}\right)$ is sub-analytic within the ball $d\left(x, x_{0}\right) \leq T$.

We extend the CC metric to a Riemannian metric and will use the notations in $\S 1.2$. So in the following $\gamma=\gamma(t), 0 \leq t \leq 1, \gamma(0)=x_{0}$, will denote a geodesic for the $\mathrm{CC}$ metric with the Lagrangian multiplier $\lambda=\lambda(t)$, which satisfy equations (1.3), (1.4).

We define a map

$$
\begin{aligned}
\exp : H_{x_{0}} \times H_{x_{0}}^{\perp} & \rightarrow M, \\
\left(\gamma^{\prime}(0), \lambda(0)\right) & \rightarrow \gamma(1)
\end{aligned}
$$

which is obviously analytic. Note this is simply the old exponential map composed with the metric-induced identification on $H_{x_{0}} \times H_{x_{0}}^{\perp}=$ $T_{x_{0}} M$ with $T_{x_{0}}^{*} M$.

LEMMA 5.4. There exists $T>0$ and a compact subset $F \subset H_{x_{0}} \times$ $H_{x_{0}}^{\perp}$ (e.g. a closed ball) such that if $\gamma:[0,1] \rightarrow M, \gamma(0)=x_{0}$, is minimizing and $d\left(\gamma(1), x_{0}\right) \leq T$, then $\left(\gamma^{\prime}(0), \lambda(0)\right) \in F$.

Proof. By contradiction.

If it is not true, then there is a sequence of minimizing geodesics $\gamma_{i}:[0,1] \rightarrow M, \gamma_{i}(0)=x_{0}, e\left(\gamma_{i}\right):=\gamma_{i}(1) \neq x_{0}$, with Lagrange multipliers $\lambda_{i}$ satisfying (1.3), (1.4), such that

$$
\gamma_{i}(1) \rightarrow x_{0}, \quad \lambda_{i}(0) \rightarrow \infty, \quad \text { as } i \rightarrow \infty .
$$

So $l_{i}=\max _{i}\left|\lambda_{i}(t)\right| \rightarrow \infty$.

Now use the coordinates of Lemma 1.1 to identify a neighborhood of $x_{0}$ with $R^{m} \times R^{k}$, where 0 corresponds to $x_{0}$ and $m$ is the distribution's rank. Then we have $\gamma_{i}=\left(\alpha_{i}, \beta_{i}\right), \alpha_{i}(t) \in R^{m}, \beta_{i}(t) \in R^{k}$. Let

$$
\delta_{i}=a\left(e\left(\gamma_{i}\right)\right)
$$


where

Define

$$
a(x)=\left(x_{i}^{4}+\cdots+x_{m}^{4}+y_{1}^{2}+\cdots+y_{k}^{2}\right)^{1 / 4} .
$$

$$
\bar{\alpha}_{i}=\frac{\alpha_{i}}{\delta_{i}}, \quad \bar{\beta}_{i}=\frac{\beta_{i}}{\delta_{i}^{2}}, \quad \bar{\gamma}_{i}=\left(\bar{\alpha}_{i}, \bar{\beta}_{i}\right) .
$$

From (1.3), we see that $\left|\gamma_{i}^{\prime}(t)\right|^{2}$ is independent of $t$. Strichartz (cf. [36], Theorem 11.1) has shown that $E\left(\gamma_{i}\right) / \delta_{i}^{2}$ is uniformly bounded. It follows that $\bar{\gamma}_{i}$ has a weakly convergent sub-sequence (still denoted by $\bar{\gamma}_{i}$ ) in $H^{1}, \bar{\gamma}_{i} \rightarrow \bar{\gamma}_{\infty}$. In particular, $\bar{\gamma}_{i} \rightarrow \bar{\gamma}_{\infty}$ uniformly in $C^{0}$. It follows from the weak convergence of $\bar{\gamma}_{i}$ that $\bar{\gamma}_{\infty}$ is a horizontal path on the tangent cone. This can be seen as follows.

As we have seen in the proof of Lemma 3.1, a horizontal path $(x, y)$ satisfies

$$
\frac{d}{d t} y_{i}(t)=\sum \Gamma_{j r}^{i} x_{j}(t) \frac{d}{d t} x_{i}(t)+\bar{T}_{i}(x, y, \dot{x}, \dot{y}), \quad i=1, \ldots, k,
$$

where $\bar{T}_{i}(x, y, z, w)$ is a smooth function

$$
\left|\bar{T}_{i}(x, y, z, w)\right| \leq C_{0}\left(|x|^{3}+|y|^{2}\right) .
$$

So

$$
\beta_{i}(t)=\int_{\alpha_{t} \mid[0, t]} \omega+O\left(\delta_{i}^{3}\right)
$$

where $\omega=\left(\omega_{1}, \ldots, \omega_{k}\right), \omega_{i}$ are as in $\S 3.3$. So it follows that

$$
\bar{\beta}_{i}(t)=\int_{\bar{\alpha}_{i} \mid[0, t]} \omega+O\left(\delta_{i}\right)
$$

i.e. the limit $\left(\bar{\alpha}_{\infty}, \bar{\beta}_{\infty}\right)$ is horizontal.

First we remark that $\bar{\gamma}_{\infty}$ is not constant, as

$$
e\left(\bar{\gamma}_{\infty}\right)=\lim \frac{e\left(\gamma_{i}\right)}{\alpha\left(e\left(\gamma_{i}\right)\right)} \neq 0
$$

From (1.4) we obtain

$$
\left|\lambda_{i}^{\prime}\right| \leq C_{0}\left(\left|\gamma_{i}^{\prime}\right|\left|\lambda_{i}\right|+\left|\gamma_{i}^{\prime}\right|^{2}\right)
$$

Hence $\lambda_{i} / l_{i}$ is uniformly bounded in $C^{0}$. So after passing to a subsequence, we assume that

$$
\frac{\lambda_{i}}{l_{i}} \rightarrow b \neq 0 \text { uniformly in } C^{0} \text {. }
$$

Dividing (1.3) by $\delta_{i}$, we obtain

$$
D_{\bar{\gamma}_{i}^{\prime}} \bar{\gamma}_{i}^{\prime}+\delta_{i} Q_{1}\left(\bar{\gamma}_{i}^{\prime}, \gamma_{i}\right)=\lambda_{i} \Omega\left(\bar{\gamma}_{i}^{\prime}+\delta_{i} Q_{2}\left(\bar{\gamma}_{i}^{\prime}, \gamma_{i}\right), \cdot\right) \text {, }
$$


where $Q_{1}$ (resp. $Q_{2}$ ) is an analytic function, quadratic (resp. linear) in the first variable. Divide (5.3) by $l_{i}$, and then taking its inner product with a $H^{1}$-map $V$ with compact support, we obtain

$$
\begin{array}{r}
\frac{1}{\bar{l}_{i}} \int_{0}^{1}\left\{-\left(\bar{\gamma}_{i}^{\prime}, V^{\prime}\right)+\left(\delta_{i} Q_{1}\left(\bar{\gamma}_{i}^{\prime}, \gamma_{i}\right), V\right)\right\} d t \\
\quad=\int_{0}^{1} \frac{\lambda_{i}}{l_{i}} \Omega\left(\bar{\gamma}_{i}^{\prime}+\delta_{i} Q_{2}\left(\bar{\gamma}_{i}^{\prime}, \gamma_{i}\right), V\right) d t .
\end{array}
$$

Let $i \rightarrow \infty$ above and note that the integral on the right-hand side is uniformly bounded to conclude that

$$
\int_{0}^{1} b \cdot \Omega\left(\bar{\gamma}_{\infty}^{\prime}, V\right) d t=0
$$

for any compactly supported $V$. But $b, \bar{\gamma}_{\infty}^{\prime} \neq 0$. This contradicts the fact that $H$ is fat. (Observe that if $H$ is fat, then so is every tangent cone.)

Proof of Theorem 4. Define two subsets

$$
\begin{gathered}
A=\left\{\left(x, t, \gamma^{\prime}(0), \lambda(0)\right) \in M \times R \times F,\right. \\
\left.x=\exp \left(\gamma^{\prime}(0), \lambda(0)\right), t=\left|\gamma^{\prime}(0)\right|\right\}, \\
B=\left\{\left(x, t, \gamma^{\prime}(0), \lambda(0)\right) \in M \times R \times F,\right. \\
\left.x=\exp \left(\gamma^{\prime}(0), \lambda(0)\right), 0<\left|\gamma^{\prime}(0)\right|<t\right\} .
\end{gathered}
$$

Since these sets are defined by analytic equations and inequalities, they are sub-analytic.

Let $P: M \times R \times F \rightarrow M \times R$ be the projection; then by definition, the graph of the distance function $t=d\left(x, x_{0}\right)$ within $d\left(x, x_{0}\right) \leq T$ is just

$$
P(A)-P(B) \text {. }
$$

Since $F$ is compact, we see that $P$ is proper on the closures of $A$ and $B$ respectively. By the definition of sub-analytic sets, $P(A)-P(B)$ is sub-analytic. So we see that the graph of $d\left(x, x_{0}\right)$ is sub-analytic, and hence $d\left(x, x_{0}\right)$ is sub-analytic within $d\left(x, x_{0}\right) \leq T$.

5.3. A stronger version of Theorem 4 . Here we will prove a stronger version of Theorem 4, using a more direct approach.

TheOREM 5.5. Suppose $H$ is fat and the CC metric on $M$ is real analytic. Choose coordinates $x=\left(x_{i}, y_{j}\right)$ near $x_{0}$ as in Lemma 1.1, and define

$$
a(x)=\left(x_{i}^{4}+\cdots+x_{m}^{4}+y_{1}^{2}+\cdots+y_{k}^{2}\right)^{1 / 4},
$$


then there is a neighborhood $U$ of $x_{0}$ such that the closure of the graph of

$$
d\left(x, x_{0}\right) / a(x)
$$

on $U-\left\{x_{0}\right\}$ is sub-analytic within $U \times R$. Moreover, when pulled back to the weighted blow-up, $d\left(x, x_{0}\right) / a(x)$ is continuous and sub-analytic.

We will need a result of Tamm [37].

LemmA 5.6. Suppose that $\varphi: N \rightarrow M$ and $g: N \rightarrow R$ are subanalytic and $\varphi$ is proper. Then the function

$$
\varphi_{g}(x)=\inf \left\{g(u): u \in \varphi^{-1}(x)\right\}
$$

is sub-analytic.

Now $d\left(x, x_{0}\right)$ can be written as

$$
d\left(x, x_{0}\right) / a(x)=\inf \{E(\gamma) / a(x), e(\gamma)=x\} .
$$

But $e$ is not proper, so Lemma 5.6 cannot be applied directly. The following result will enable us to overcome this difficulty.

LEMMA 5.7 ([17]). Let $B$ be a Hilbert space, $f(y, z): B \times R^{r} \rightarrow R$ an analytic function satisfying

$$
\left(D_{y}^{2} f(0,0) a, b\right)=((I-A) a, b), \quad a, b \in B,
$$

where $A$ is a linear compact operator of $B, B=\operatorname{ker}(I-A) \oplus V$. Then there are local origin preserving analytic transformations $T: B \times R^{r} \rightarrow$ $B \times R^{r}$ and $h: \operatorname{ker}(I-A) \times R^{r} \rightarrow B$, defined in a neighborhood of $(0,0) \in \operatorname{ker}(I-A) \times R^{r}$, such that $f$ takes the form of

$$
\begin{aligned}
f \circ T\left(\left(y_{1}, y_{2}\right) z\right)=\left(\left(I-A_{1}\right) y_{2}, y_{2}\right) & +f\left(h\left(y_{1}, z\right), z\right), \\
y & =\left(y_{1}, y_{2}\right) \in \operatorname{ker}(I-A) \oplus V,
\end{aligned}
$$

where $A_{1}$ is the restriction of $A$ to $V$.

In the proof of Theorem 5.5 we will use the resolution of $e$, as in Lemma 5.7 it requires that the projection is a submersion (recall that $e$ is not a submersion). 
Proof of Theorem 5.5. Write

$$
\begin{aligned}
& \frac{1}{a\left(B_{w}(z)\right)} d\left(B_{w}(z), x_{0}\right) \\
& =\inf \left\{\frac{B^{*} E(\gamma)^{1 / 2}}{a\left(B_{w}(z)\right)}, \gamma \in B\left(\Omega_{H} M\left(x_{0}, \cdot\right)-\Omega_{H} M\left(x_{0}, x_{0}\right)\right),\right. \\
& \left.B_{e}(\gamma)=z\right\} .
\end{aligned}
$$

We note that the functional in (5.6), $B^{*} E(\gamma)^{1 / 2} / a\left(B_{w}(z)\right)$, has an analytic continuation to the boundary of the domain. In fact, we have

$$
E(a \bar{\gamma})=a^{2} E_{0}(\bar{\gamma})+a^{3} E_{1}(\bar{\gamma}, a),
$$

where $E_{0}$ is the energy functional on the tangent cone, $E_{1}$ an analytic functional. So

$$
\begin{aligned}
\frac{B^{*} E(\gamma)}{a\left(B_{w}(z)\right)^{2}}= & E_{0}\left(\frac{B(\gamma)}{a\left(B_{w} \circ B_{e}(\gamma)\right)}\right) \\
& +a\left(B_{w} \circ B_{e}(\gamma)\right) E_{1}\left(\frac{B(\gamma)}{a\left(B_{w} \circ B_{e}(\gamma)\right)}, a\left(B_{w} \circ B_{e}(\gamma)\right)\right) .
\end{aligned}
$$

Since all the terms above are analytic, so the functional in (5.6) has an analytic continuation. Moreover, when $z \in B_{w}^{-1}\left(x_{0}\right)$, the minimizers of (5.6) come from the minimizing geodesics on the tangent cone $N$.

We first check that the condition in Lemma 5.7 is satisfied when $z \in B_{w}^{-1}\left(x_{0}\right)$. From (5.7), we see that the second derivative of $E_{0}$ is associated with the Jacobi's field on the tangent cone $N$, which is a 2nd order linear differential-integral equation (cf. [12], page 93)

$$
D^{2}-L=0 \text {, }
$$

where $L$ is a 1 st order linear differential-integral operator. So

$$
A=D^{-2} L
$$

and hence $A$ is compact.

Consider the set of minimizing geodesics $\gamma$ on the tangent cone, with $e(\gamma) \neq 0 . \quad R_{+}$acts on this sets, induced from its action on $N$. Let $S$ be the quotient of this set by the action of $R_{+}$, then $S$ is compact. Then, by (5.7), the set of minimizers of (5.6) when $z \in B_{w}^{-1}\left(x_{0}\right)$ is just $S$. Thus every point in $S$ has a neighborhood in $B\left(\Omega_{H} M\left(x_{0}, \cdot\right)-\Omega_{H} M\left(x_{0}, x_{0}\right)\right)$ in which Lemma 5.6 is valid. Choose a cover of $S$ by a finite number of such neighborhoods, denoted by 
$U_{i}$ (such a cover exists since $S$ is compact). If we can prove that the minimizers of (5.6) are contained in a compact subset in $\bigcup U_{i}$ if $z$ is sufficiently near $x_{0}$, then we can use Lemma 5.6 and Lemma 5.7 to prove the theorem.

Then we only need a corollary of Lemma 5.4 (whose proof is rather straight forward) to complete the proof.

COROLlary 5.8. There is an open set $W \subset \cup U_{i}$ such that $B_{e}(W)$ contains an open neighborhood of $x_{0}$, and if $z \in B_{e}(W)$, then the minimizers of (5.6) lie in $W$.

REMARK. The cut-locus of a CC metric seems hopelessly complicated in general, despite Theorem 4. We don't even know what a cut-locus in a general three-dimensional CC manifold looks like diffeomorphically, while in Riemannian geometry, the generic cut-locus on manifolds of dimension $\leq 6$ admits a complete classification up to diffeomorphisms (Bunchner [8]). Even the case of a left invariant $\mathrm{CC}$ metric on a nilpotent group is not trivial, as the energy functional here has non-isolated critical points (cf. $\S 6$ ). Montgomery has found complicated "secondary conjugate locus" on Heisenberg groups. We conjecture that if the cut locus of any point of a three-dimensional fat CC manifold is locally diffeomorphic to a line, then the metric is homogeneous, and for every point there is a $S^{1}$ isometric group which leave both this point and the cut-locus through this point invariant.

6. Left-invariant $\mathrm{CC}$ metrics on fat two-step nilpotent groups. As we have shown in previous sections, the geometry of a CC manifold $M$ is largely determined by the tangent cones, especially when $H$ is fat. In this section we will study the CC geometry on fat nilpotent group.

6.1. The case of codimension 1. Let $n=V_{1} \oplus V_{2}$ be a fat twostep nilpotent Lie algebra, $\left[V_{1}, V_{1}\right]=V_{2},\left[V_{1}, V_{2}\right]=\left[V_{2}, V_{2}\right]=0$, $\operatorname{dim} V_{1}=m, \operatorname{dim} V_{2}=k$, and $N$ be the corresponding simply connected nilpotent group.

We begin with the case $k=1$. The nilpotent Lie algebra then is Heisenberg. We first look at the case $n=3$ which was studied in detail in [11]. Take $x, y, z$ as the coordinates on $N$ such that $H$ is spanned by $\partial / \partial x-y \partial / \partial z, \partial / \partial y+x \partial / \partial z$, and the CC metric is (up to a constant) $Q=(d x-y d z)^{2}+(d y+x d z)^{2}$. The geodesic flow on $T^{*} H$ passing through $x=y=z=0$ is 


$$
\begin{aligned}
(x(t), y(t), z(t) & , p(t), q(t), r(t)), \text { where } \\
r(t) & =r(0)=r, \\
x(t) & =\sin (4 r t)(p(0) \cos (4 r t)+q(0) \sin (4 r t)) / 2 r, \\
y(t) & =\sin (4 r t)(-p(0) \sin (4 r t)+q(0) \cos (4 r t)) / 2 r, \\
p(t) & =\cos (4 r t)(p(0) \cos (4 r t)+q(0)) \sin (4 r t), \\
q(t) & =\cos (4 r t)(-p(0) \sin (4 r t)+q(0)) \cos (4 r t), \\
z(t) & =2 r^{-1}\left(t-\frac{\sin (8 r t)}{8 r}\right)\left(p^{2}(0)+q^{2}(0)\right) .
\end{aligned}
$$

The 1st conjugate locus of $(0,0,0)$ is the set of points $(0,0, z)$. Moreover, the Morse index of $(0,0, z)(z \neq 0)$ along a prime geodesic (i.e. a geodesic which does not contain conjugate point except the end point) is 1 . In this case, the 1 st conjugate locus coincides with the cut locus.

If $n \geq 5$, the CC metric can be written as

$$
\sum a_{i}\left(\left(d x_{i}-y_{i} d z\right)^{2}+\left(d y_{i}+x_{i} d z\right)^{2}\right),
$$

where $a_{i}$ are positive numbers. It is easy to prove the following result:

Lemma 6.1. A point $(0,0, z)$ is a conjugate point of $(0,0,0)$. If $a_{i} \neq a_{j}$ for $i \neq j$, the Morse index of $(0,0, z)(z \neq 0)$ to $(0, \ldots, 0,0)$ along a prime geodesic is 1 , while if $a_{1}=a_{2}=\cdots=a_{t}$, the Morse index is $t(t-1) / 2 \quad(n=2 t+1)$.

In this case, if $a_{1}=a_{2}=\cdots=a_{t}$, then the cut-locus of 0 is the set $\{(0, \ldots, 0, z)\}$; otherwise the cut locus is rather complicated.

6.2. The case $k=2$. In this case the fat two-step nilpotent Lie algebra is not unique for given $n$. Nevertheless, we have the following result.

LEMMA 6.2. The set of fat two-step nilpotent Lie algebras with $m=$ $n-2$ is connected for given $n$.

Thus the "moduli space" of fat CC metrics with $k=2$ is connected. To prove this lemma, we need the following results.

LEMMA 6.3. The set

$$
U=\left\{A \in \mathrm{GL}(n, R), J_{n} A=A^{\top} J_{n} A \text {, has no real eigenvalues }\right\}
$$


is connected. Here $J_{n}$ is the matrix

$$
J_{n}=\left(\begin{array}{cc}
0 & I_{n} \\
-I_{n} & 0
\end{array}\right)
$$

Proof. Any element $A$ in $U$ can be written as

$$
P A P^{-1}=\left(\begin{array}{cc}
B & 0 \\
0 & \bar{B}
\end{array}\right) \text {, }
$$

where $B$ is a complex matrix, $\bar{B}$ its complex conjugation, $B \neq \bar{B}, P$ is an invertible matrix of the form of

$$
P=\left(\begin{array}{ll}
P_{1} & \bar{P}_{1} \\
P_{2} & \bar{P}_{2}
\end{array}\right) \text {. }
$$

From $J A=A^{\top} J$, we see that $P$ must satisfy

$$
P_{1}^{\top} \bar{P}_{2}-P_{2}^{\top} \bar{P}_{1}=0
$$

i.e. $P_{1}^{\top} \bar{P}_{2} \in u(t, C)$ (the space of $t \times t$ infinitesimally hermitian matrices, $n=2 t)$. Such $P$ is called admissible. We want to prove that the set of admissible $P$ is connected.

(1) If $P_{1}$ is non-singular, then $P$ can be connected to

$$
\left(\begin{array}{cc}
I & I \\
P_{2} P_{1}^{-1} & \bar{P}_{2} \bar{P}_{2}^{1}
\end{array}\right)
$$

by a curve of admissible $P_{t}$

$$
\left(\begin{array}{ll}
P_{1} A_{t} & \bar{P}_{1} \bar{A}_{t} \\
P_{2} A_{t} & \bar{P}_{2} \bar{A}_{t}
\end{array}\right)
$$

where $A_{t}$ is a matrix depending continuously on $t, A_{0}=I, A_{1}=$ $P_{1}^{-1}$.

(2) If $P_{1}$ is singular, then there are $C, D \in \mathrm{GL}(t, C)$ such that $C^{\top} \bar{P}_{2} \bar{D} \in u(t, C)$. Since for any $D,\left(P_{1} D\right)^{\top}\left(\bar{P}_{2} \bar{D}\right) \in u(t, C)$ whenever $P_{1}^{\top} \bar{P}_{2} \in u(t, C)$, hence $P$ can be connected to

$$
\left(\begin{array}{ll}
W_{1} & \bar{W}_{1} \\
W_{2} & \bar{W}_{2}
\end{array}\right)=\left(\begin{array}{ll}
P_{1} & \bar{P}_{2} \\
P_{2} & \bar{P}_{2}
\end{array}\right)\left(\begin{array}{cc}
D & 0 \\
0 & \bar{D}
\end{array}\right)
$$

and then to matrix

$$
\left(\begin{array}{cc}
W_{1}+t C & \bar{W}_{1}+t \bar{C} \\
W_{2} & \bar{W}_{2}
\end{array}\right) .
$$

Note that for $t$ small enough $W_{1}+t C$ is non-singular. Thus we have reduced the case (2) to the case (1). 
So far we have proved that any $P$ can be connected to a matrix of the form

$$
\left(\begin{array}{ll}
I & I \\
F & \bar{F}
\end{array}\right)
$$

where $F \in u(t, C), \operatorname{det}(F-\bar{F}) \neq 0$. On the other hand, the set

$$
\{F \in u(t, C), \operatorname{det}(F-\bar{F}) \neq 0\}
$$

is contractible to the set of non-singular anti-symmetric matrices, which is connected. So, the set of admissible $P$ is connected.

Having proved that the set of admissible $P$ is connected, we can prove that the set $U$ is connected. In fact, let $A$ be any matrix of the form (6.1) with $B \neq \bar{B}$; then there is $B_{t}$ such that $B_{0}=B, B_{1}=i I_{t}$, $\bar{B}_{t} \neq B_{t}$, and depends continuously on $t$. So any $A \in U$ can be connected in $U$ to

$$
P\left(\begin{array}{cc}
i I & 0 \\
0 & -i I
\end{array}\right) P^{-1}
$$

where $P$ is admissible, which forms a connected set, since the set of admissible $P$ is connected.

Proof of Lemma 6.2. Suppose that the two-step nilpotent Lie algebra is of the form of $R^{m}+R^{2}$ with $\left[R^{m}, R^{m}\right]=R^{2},\left[R^{m}, R^{2}\right]=$ $\left[R^{2}, R^{2}\right]=0$. Write $[\cdot, \cdot]: R^{m} \times R^{m} \rightarrow R^{2}$ as $[a, b]=\left(a^{\top} B_{1} b, a^{\top} B_{2} b\right)$, where $B_{1}, B_{2}$ are skew-symmetric matrices. The Lie algebra is fat if and only if for every $\left(c_{1}, c_{2}\right) \in R^{2}-\{0\}$,

$$
\operatorname{det}\left(c_{1} B_{1}+c_{2} B_{2}\right) \neq 0 \text {. }
$$

We denote the set of such pairs $\left(B_{1}, B_{2}\right)$ by $V$. Write $B_{1}$ as $B_{1}=$ $P^{\top} J P$, with $P$ invertible. Write $B_{2}=P^{\top} D P$; then (6.2) is equivalent to $\operatorname{det}\left(c_{1} J+c_{2} D\right) \neq 0$. Denote the set of skew-matrices $D$ satisfying the above condition by $U_{1}$. Then $V$ is connected if and only if $U_{1}$ is. So we need only to prove that $U_{1}$ is connected. But $U_{1}$ is precisely the set $U$ in Lemma 6.3 , since the map $U_{1} \rightarrow U$ which sends $D$ to $D J^{-1}$ is a homeomorphism.

6.3. Generic cases. We will study general left-invariant CC metrics when $N-m \geq 2$.

Given a fat nilpotent Lie algebra $V_{1}+V_{2},\left[V_{1}, V_{1}\right]=V_{2},\left[V_{1}, V_{2}\right]=$ 0 , denote the $V_{2}$-valued anti-symmetric bilinear form on $V_{1}, J\left(a_{1}, a_{2}\right)$ $=\left[a_{1}, a_{2}\right]$. Then the multiplication of the Lie group is $\left(a_{1}, b_{1}\right)\left(a_{2}, b_{2}\right)$ 
$=\left(a_{1}+a_{2}, b_{1}+b_{2}+J\left(a_{1}, a_{2}\right) / 2\right)$. Let $L$ be the left translation. Then

$$
\begin{aligned}
T L_{\left(a_{1}, b_{1}\right)}(\delta a, \delta b) & =\left(\delta a, \delta b+J\left(a_{1}, \delta a\right) / 2\right), \\
L_{\left(a_{1}, b_{1}\right)}^{*}(d a, d b) & =\left(d a+J^{*}\left(a_{1}, d b\right) / 2, d b\right),
\end{aligned}
$$

where $J^{*}: V_{1} \times V_{2}^{*} \rightarrow V_{1}^{*}$ is defined by

$$
\left(J^{*}(a, b)\right)(c)=b(J(a, c)), \quad a, c \in V_{1}, b \in V_{2}^{*}
$$

For a metric $Q_{0}$ on $V_{1}^{*}$, the induced left-invariant CC metrices is $Q_{0}\left(d a+J^{*}(a, d b)\right)$. We identify $V_{1}$ with $V_{1}^{*}$ via $Q_{0}$. The Hamiltonian of the Carnot-Carathéodory metric is

$$
Q_{0}\left(p+J^{*}(a, r)\right), \quad(a, b, p, r) \in T^{*} N
$$

Since (6.3) does not contain $b$ explicitly, $r$ is a $V_{2}^{*}$-valued first integral of the Hamiltonian (6.3).

Fixing $r_{0} \in V_{2}^{*}$, define $J_{r_{0}}: V_{1} \rightarrow V_{1}$ by $J_{r_{0}}(a)=J^{*}\left(a, r_{0}\right)$. Then the Hamiltonian system (6.3) can be written as

$$
\frac{d}{d t}\left(\begin{array}{l}
a \\
p
\end{array}\right)=\left(\begin{array}{cc}
J_{r_{0}} & I \\
-J_{r_{0}}^{*} J_{r_{0}} & -J_{r_{0}}^{*}
\end{array}\right)\left(\begin{array}{l}
a \\
b
\end{array}\right) .
$$

Let $P$ be an orthogonal matrix such that

$$
J_{r_{0}}=P \quad \operatorname{diag}\left(c_{1} J_{2}, \ldots, c_{t} J_{2}\right) P^{-1},
$$

where $\pm c_{1} i, \pm c_{2} i, \ldots, \pm c_{t} i$ are the eigenvalues of $J_{r_{0}}, c_{i}>0$. Intrcduce $a=P \bar{a}, p=P^{-1} \bar{p}$; then (6.4) can be rewritten as

$$
\frac{d}{d t}\left(\begin{array}{l}
\bar{a} \\
\bar{p}
\end{array}\right)\left(\begin{array}{cc}
\operatorname{diag}\left(c_{1} J_{2}, \ldots, c_{t} J_{2}\right) & I \\
-\operatorname{diag}\left(c_{1}^{2} I_{2}, \ldots, c_{t}^{2} I_{2}\right) & -\operatorname{diag}\left(c_{1} J_{2}, \ldots, c_{t} J_{2}\right)
\end{array}\right)\left(\begin{array}{l}
\bar{a} \\
\bar{p}
\end{array}\right)
$$

Write $\bar{a}$ as $\left(\bar{a}_{1}, \ldots, \bar{a}_{t}\right)$ and similarly for $\bar{p}$. Then (6.4) can be written as

$$
\frac{d}{d t}\left(\begin{array}{c}
\bar{a}_{i} \\
\bar{p}_{i}
\end{array}\right)=\left(\begin{array}{cc}
c_{i} J_{2} & I \\
-c_{i}^{2} I_{2} & -c_{i} I_{2}
\end{array}\right)\left(\begin{array}{c}
\bar{a}_{i} \\
\bar{p}_{i}
\end{array}\right), \quad i=1, \ldots, t
$$

which has been solved in $\S 6.1$. 
LEMMA 6.4. If the eigenvalues of $J_{r_{0}}$ are $\pm c_{1} i, \ldots, \pm c_{t} i ; c_{i}>0$, $c_{i} \neq c_{j}$, for $i \neq j$, then the Morse index of $(0, z)$ to $(0,0)$ along $a$ prime geodesic $c$ for which $r(c)=r_{0}$ is 1 .

REMARK 1. In general the cut-locus is rather complicated.

REMARK 2. It seems that a "generic" conjugate point has multiplicity 1 . More precisely, we conjecture that the following is true: Fixing $m, n$ with $n \geq 4$, then given a positive definite bilinear form $Q_{0}$ on $V_{1}^{*}$, there is an open and dense set of nilpotent Lie algebras such that a generic conjugate point of $0 \in V_{1} \times V_{2}$ of the induced fat left-invariant $C C$ metric has multiplicity 1.

\section{REFERENCES}

[1] V. I. Arnold, S. M. Gusein-Zade and A. N. Varchenko, Singularities of differentiable maps, vol. 1, Birkhäuser, Boston, 1985.

[2] M. Atiyah and A. Pressley, Convexity and loop groups, Progr. Math., vol. 36, 33-64, Birkhäuser, Basel, 1983.

[3] C. Bär, Geodesics for Carnot-Carathéodory metrices, preprint, 1989.

[4] G. Ben Arous, Noyau de la chaleur hypoelliptique et geometrie sous-riemannienne, in Stochastic Analysis, M. Metivier and S. Watanabe eds., Lecture Notes in Math., vol. 1322, Springer-Verlag, 1988.

[5] L. Bergery, Sur certanies fibrations d'espaces homogenes riemannies, Compositio Math., (1975), 43-61.

[6] J. M. Bismut, Large derivations and the Malliavin calculus, Progr. Math., vol. 45, Birkhäuser, Basel, 1984.

[7] R. Brockett, Control Theory and Singular Riemannian Geometry, in New Directions in Applied Mathematics, Springer-Verlag, New York, 1981.

[8] M. A. Buchner, Stability of the cut-locus in dimensions less than or equal to six, Invent. Math., 43 (1977), 199-237.

[9] W. L. Chow, Uber systeme von linearen partiellen differentialgleichungen erster order, Math. Ann., 117 (1939), 98-105.

[10] D. Freed, Geometry of the loop group, J. Differential Geom., 28 (1988), 223276.

[11] B. Gaveau, Principle de moindre action, propagation de la challeur et estimates sour-elliptic sur certains grouppes nilpotent, Acta Math., 139 (1977), 85-153.

[12] Zhong Ge, On a variational problem and the spaces of horizontal paths, Pacific J. Math., 149 (1991), 61-93.

[13] E. Getzler, Dirichlet forms on loop spaces, Bull. Sci. Math., $2^{\mathrm{e}}$ serie, 113 (1989), 151-174.

[14] R. W. Goodman, Nilpotent Groups, the Structure, and the Application to Analysis, Lecture Notes in Math., vol. 562, Springer, 1977.

[15] D. Gromoll and W. Meyer, On differentiable functions with isolated critical points, Topology, 18 (1969), 361-369.

[16] M. Gromov, Sur structure metriques pour les varieties riemannian, CedicNathan. 
[17] Groups of polynomial growth and expanding maps, Inst. Hautes Sci. Publ. Math., 53 (1981).

[18] U. Hammenstädt, Zur theorie von Carnot-Carathéodory-metriken und ihren anwendungen doktorarbeit, Bonn 1986.

[19] __ Some regularity in Carnot-Carathéodory metrics, J. Differential Geom., 32 (1991), 192-201.

[20] R. Hermann, Some differential-geometric aspects of the Lagrangian variational problem, Illinois J. Math., 6 (1962), 634-673.

[21] S. Kobayashi, On conjugate and cut loci, in Studies in Global Geometry and Analysis, S. S. Chern ed., Mathematical Association of America, New Jersey, 1967.

[22] A. Koranyi, Geometric properties of Heisenberg-type group, Adv. in Math., 56 (1985), 28-38.

[23] A. Koranyi and H. M. Riemann, Quasiconformal mappings on the Heisenberg group, Invent. Math., 80 (1985), 309-338.

[24] J. Milnor, Morse theory, Ann. of Math. Studies, vol. 51, Princeton, N.J., 1963.

[25] - Singularity on the complex hyperplane, Ann. of Math. Studies, vol. 61, Princeton, N.J., 1968.

[26] J. Mitchell, On Carnot-Carathéodory metrics, J. Differential Geom., 21 (1985), 35-45.

[27] R. Montgomery, The isoholonomic problem and some applications, Comm. Math. Phys., 128 (1990), 565-592.

[28] - The geodesics which do not satisfy the geodesic equations, preprint.

[29] P. Orlik and P. Wagreigh, Isolated singularities of algebraic surfaces with $C^{*}$ action, Ann. of Math., 93 (1971), 205-228.

[30] P. Pansu, Croissance des boules et quasiisometries dans les nilvarieties, Ergodic Theory Dynamical Systems, 3 (1983), 441-443.

[31] _. Metriques de Carnot-Carthéodory et quasiisometries des espaces symetriques de rang an, Ann. of Math., 129 (1989), 441-443.

[32] C. B. Rayer, The exponential map for the Lagrange problem on differentiable manifold, Phil. Trans. Roy. Soc. London, A, Math. and Phys., 1127 (1967), 299-344.

[33] L. Rothschild and E. Stein, Hypoelliptic differential operators and nilpotent groups, Acta Math., 137 (1976), 247-320.

[34] S. Smale, Regular curves on Riemannian manifold, Trans. Amer. Math. Soc., 87 (1958), 446-462.

[35] E. H. Spanier, Algebraic Topology, McGraw-Hill, New York, 1986.

[36] R. S. Strichartz, Sub-riemannian geometry, J. Differential Geom., 24 (1986), 221-263.

[37] M. Tamm, Sub-analytic sets in the calculus of variations, Acta Math., 146 (1981), 167-199.

[38] T. Taylor, Some aspects of differential geometry associated with hypoelliptic second order operators, Pacific J. Math., 136 (1989), 355-378.

[39] A. M. Vershik and C. Ya. Gershkovich, Non-holonomic manifolds and nilpotent analysis, J. Geom. Phys., 5 (1988), no. 3.

[40] S. Webster, Pseudo-hermitian structures on a real hyperplane, J. Differential Geom., 13 (1978), 25-40.

[41] A. Weinstein, Fat bundles and symplectic manifolds, Adv. in Math., 37 (1980), 239-250.

[42] H. Sussmann, Subanalyticity of the distance functions for real analytic subRiemannian metrics on three dimensional manifolds, Report SYCON-91-05a, Rutgers, 1991. 
[43] S. Kobayashi and K. Nomizu, Foundation of Differential Geometry, vol. 2, Interscience, 1963.

[44] A. Kupka, The ubiquity of Fuller's phenomenon, in Nonlinear Controllablity and Optimal Control, Sussmann ed., Marcel Dekker, 1990, 313-350.

Received April 24, 1991 and in revised form June 22, 1992. This work was supported in part by the National Sciences Foundation of China for Young Scientists.

The Fields Institute

185 Columbia St. West

WATERLOO, ONTARIO, N2L 5Z5

CANADA 


\title{
PACIFIC JOURNAL OF MATHEMATICS
}

Founded by

\author{
E. F. BECKenBaCH (1906-1982) F. WolF (1904-1989)
}

\section{EDITORS}

Sun-Yung A. Chang

(Managing Editor)

University of California

Los Angeles, CA 90024-1555

chang@math.ucla.edu

F. Michael Christ

University of California

Los Angeles, CA 90024-1555

christ@math.ucla.edu

Herbert Clemens

University of Utah

Salt Lake City, UT 84112

clemens@math.utah.edu

\author{
R. FInN
}

THOMAS ENRIGHT

University of California, San Diego

La Jolla, CA 92093

tenright@ucsd.edu

Nicholas ERColani

University of Arizona

Tucson, AZ 85721

ercolani@math.arizona.edu

Stanford University

Stanford, CA 94305

finn@gauss.stanford.edu

VAUghan F. R. JoNES

University of California

Berkeley, CA 94720

vfr@math.berkeley.edu

\section{SUPPORTING INSTITUTIONS}

STEVEN KeRCKHOFF

Stanford University

Stanford, CA 94305

spk@gauss.stanford.edu

MARTIN ScharLemanN

University of California

Santa Barbara, CA 93106

mgscharl@henri.ucsb.edu

HAROLd STARK

University of California, San Diego La Jolla, CA 92093

V. S. VARADARAJAN

University of California

Los Angeles, CA 90024-1555

vsv@math.ucla.edu

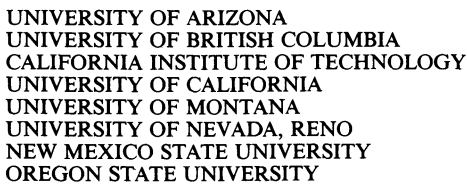

The Supporting Institutions listed above contribute to the cost of publication of this Journal, but they are not owners or publishers and have no responsibility for its content or policies.

Mathematical papers intended for publication in the Pacific Journal of Mathematics should be in typed form or offset-reproduced (not dittoed), double spaced with large margins. Please do not use built up fractions in the text of the manuscript. However, you may use them in the displayed equations. Underline Greek letters in red, German in green, and script in blue. The first paragraph must be capable of being used separately as a synopsis of the entire paper. In particular it should contain no bibliographic references. Please propose a heading for the odd numbered pages of less than 35 characters. Manuscripts, in triplicate, may be sent to any one of the editors. Please classify according to the 1991 Mathematics Subject Classification scheme which can be found in the December index volumes of Mathematical Reviews. Supply name and address of author to whom proofs should be sent. All other communications should be addressed to the managing editor, or Julie Honig, University of California, Los Angeles, California 90024-1555.

There are page-charges associated with articles appearing in the Pacific Journal of Mathematics. These charges are expected to be paid by the author's University, Government Agency or Company. If the author or authors do not have access to such Institutional support these charges are waived. Single authors will receive 75 free reprints; joint authors will receive a total of 100 free reprints. Additional copies may be obtained at cost in multiples of 50 .

The Pacific Journal of Mathematics (ISSN 0030-8730) is published monthly except for July and August. Regular subscription rate: $\$ 200.00$ a year (10 issues). Special rate: $\$ 100.00$ a year to individual members of supporting institutions.

Subscriptions, orders for numbers issued in the last three calendar years, and changes of address should be sent to Pacific Journal of Mathematics, P.O. Box 969, Carmel Valley, CA 93924, U.S.A. Old back numbers obtainable from Kraus Periodicals Co., Route 100, Millwood, NY 10546.

The Pacific Journal of Mathematics at P.O. Box 969, Carmel Valley, CA 93924 (ISSN 0030-8730) is published monthly except for July and August. Second-class postage paid at Carmel Valley, California 93924, and additional mailing offices. Postmaster: send address changes to Pacific Journal of Mathematics, P.O. Box 969, Carmel Valley, CA 93924.

PUBLISHED BY PACIFIC JOURNAL OF MATHEMATICS, A NON-PROFIT CORPORATION

This publication was typeset using $\mathcal{A} \mathcal{M S}-\mathrm{T}_{\mathrm{E}} \mathrm{X}$, the American Mathematical Society's $\mathrm{T}_{\mathrm{E}} \mathrm{X}$ macro system. Copyright (c) 1993 by Pacific Journal of Mathematics 


\section{PACIFIC JOURNAL OF MATHEMATICS}

Volume $161 \quad$ No. $2 \quad$ December 1993

On the method of constructing irreducible finite index subfactors of 201 Popa

FLORIN PETRE BOCA

Brownian motion and the heat semigroup on the path space of a

233 compact Lie group

JAY BARRY EPPERSON and TERRY M. LOHRENZ

Horizontal path spaces and Carnot-Carathéodory metrics

255

ZHONG GE

Biholomorphic convex mappings of ball in $\mathbb{C}^{n}$

SHENG GONG, SHI KUN WANG and QI HUANG YU

The Temperley-Lieb algebra at roots of unity

FREDERICK MiCHAEL GOODMAN and HANS WENZL

Jordan analogs of the Burnside and Jacobson density theorems

LUZIUS GRÜNENFELDER, M. OLMLADIČ and HEYDAR RADJAVI

Elliptic representations for $\mathrm{Sp}(2 n)$ and $\mathrm{SO}(n)$

REBECCA A. Herb

Reflexivity of subnormal operators

JOHN MCCARTHY

Knotting trivial knots and resulting knot types

KiMiHiko MOTEGI

Commutativity of selfadjoint operators

MitsURU UCHIYAMA

Correction to: "One-dimensional Nash groups" 